\title{
$\Rightarrow$ \\ Not so local: the population genetics of convergent adaptation in maize and teosinte.
}

\author{
Silas Tittes ${ }^{a, b}, 1$, Anne Lorant ${ }^{c}$, Sean McGintyc ${ }^{c}$ John F. Doebley ${ }^{\mathrm{d}}$, James B. Holland ${ }^{\mathrm{e}}$, Jose de Jesus Sánchez-González ${ }^{\mathrm{f}}$, Arun \\ Seetharamg, Maud Tenaillon ${ }^{\mathrm{h}}$ and Jeffrey Ross-lbarra ${ }^{\mathrm{a}, \mathrm{b}, \mathrm{i}, 1}$ \\ a Dept. of Evolution and Ecology, University of California, Davis, CA 95616, USA, benter for Population Biology, University of California, Davis, CA 95616, USA, \\ 'Department of Plant Sciences, University of California, Davis, CA 95616, USA, ${ }^{\mathrm{d}}$ Laboratory of Genetics, University of Wisconsin - Madison, Madison, WI \\ 53706 USA, eUnited States Department of Agriculture - Agriculture Research Service, Raleigh, NC 27695 USA and Department of Crop and Soil Sciences, \\ North Carolina State University, ${ }^{\mathrm{f} C e n t r o ~ U n i v e r s i t a r i o ~ d e ~ C i e n c i a s ~ B i o l o ́ g i c a s ~ y ~ A g r o p e c u a r i a s, ~ U n i v e r s i d a d ~ d e ~ G u a d a l a j a r a, ~ Z a p o p a n, ~ J a l i s c o ~ C P 45110, ~ M e ́ x i c o, ~}$ \\ gDepartment of Ecology, Evolution, and Organismal Biology; Genome Informatics Facility, lowa State University, Ames, IA 50011, USA, haénétique \\ Quantitative et Evolution - Le Moulon, Université Paris-Saclay, INRAE, CNRS, AgroParisTech, 91190, Gif-sur-Yvette, France, ${ }^{\mathrm{i} G e n o m e ~ C e n t e r, ~ U n i v e r s i t y ~ o f ~}$
} California, Davis, CA 95616, USA

\begin{abstract}
What is the genetic architecture of local adaptation and what is the geographic scale that it operates over? We investigated patterns of local and convergent adaptation in five sympatric population pairs of traditionally cultivated maize and its wild relative teosinte (Zea mays subsp. parviglumis). We found that signatures of local adaptation based on the inference of adaptive fixations and selective sweeps are frequently exclusive to individual populations, more so in teosinte compared to maize. However, for both maize and teosinte, selective sweeps are frequently shared by several populations, and often between the subspecies. We were further able to infer that selective sweeps were shared among populations most often via migration, though sharing via standing variation was also common. Our analyses suggest that teosinte has been a continued source of beneficial alleles for maize, post domestication, and that maize populations have facilitated adaptation in teosinte by moving beneficial alleles across the landscape. Taken together, out results suggest local adaptation in maize and teosinte has an intermediate geographic scale, one that is larger than individual populations, but smaller than the species range.
\end{abstract}

KEYWORDS Local Adaptation, Convergent Adaptation, Maize, Teosinte

\section{Introduction}

As populations diverge and occupy new regions, they become locally adapted to the novel ecological conditions that they encounter. Decades of empirical work have carefully documented evidence for local adaptation, including the use of common garden and reciprocal transplant studies demonstrating that populations express higher fitness in their home environment (Clausen et al. 1948) as well as quantitative genetic approaches that show selection has acted on individual traits to make organisms better suited to their ecological conditions (Savolainen et al. 2013). It is clear from these studies that local adaptation is pervasive in natural populations.

One important but understudied aspect of local adaptation is its geographic scale. Empirical studies have documented adaptation at multiple scales, from microgeographic differentiation among mesic and xeric habitats along a single hillside (Hamrick and Allard 1972) to regional (Lowry et al. 2008; Whitehead et al. 2011) and even global scales (Colosimo et al. 2005). A key factor determining the geographic scale of local adaptation is the distribution of the biotic and abiotic challenges to which organisms are adapting, as these features place limits on the

${ }^{1}$ For correspondence, Dept. of Evolution and Ecology, University of California, Davis,
CA, USA E-mail: stittes@ucdavis.edu; rossibarra@ucdavis.edu locations over which an allele remains beneficial. Environmental features overlap with each other to varying degrees (Tuanmu and Jetz 2015). The degree of overlap between environmental features may be important if mutations are pleiotropic, as an allele may not be beneficial when integrating its effect over multiple selective pressures (Chevin et al. 2010).

The geographic scale of local adaptation depends, too, on population structure. Gossmann et al. (2010) showed that the estimates of the proportion of new mutations fixed by natural selection across a number of plant species tended to overlap with zero, suggesting there is little evidence for adaptation at non-synonymous sites. One potential explanation raised by the authors for this surprising finding was that natural populations are often structured, such that very few adaptations would be expected to be common over the entire range of the species' distribution. Indeed, even when selective pressures are shared across populations, structure can hinder a species' adaptation by limiting the spread of beneficial alleles across the species range (Bourne et al. 2014). Consistent with this, Fournier-Level et al. (2011) conducted a continent-scale survey across strongly structured populations of Arabidopsis thaliana, finding that alleles which increase fitness tended to occur over a restricted geographic scale. Despite the progress made, it remains unclear if the scales identified in Arabidopsis are common to local adaptation across the entire genome, and how similar the general 
patterns are across taxa.

The majority of local adaptation studies are motivated by con- 111 spicuous differences in the phenotypes or environments of two 112 or more populations. As such, many instances of local adapta- ${ }_{113}$ tion that are occurring, as well as the underlying beneficial muta- 114 tions being selected, may go overlooked. This hinders our ability ${ }^{115}$ to draw more general conclusions about the overall frequency 116 and impact of local adaptation on a given population's evolu- 117 tionary history. Efforts to more systematically survey evidence 118 of local adaptation have led to "reverse ecology" approaches, 119 where signatures of adaptive evolution are first identified from 120 genomic sequencing data, and are then related to the traits, his- 121 tory, or environmental conditions of the populations in which 122 they occur (Li et al. 2008; Levy and Borenstein 2012). Given their ${ }_{123}$ ability to survey numerous loci and populations simultaneously, 124 reverse ecology and other population genetic approaches are ${ }^{125}$ well-suited to investigating the geographic scale of local adapta- 126 tion. While the use of genome-scale reverse ecology approaches 127 is increasing (Hoban et al. 2016; Bragg et al. 2015), sampling ${ }^{12}$ across multiple populations and explicit evaluation of the scale ${ }^{129}$ of local adaptation are still uncommon.

Using reverse ecology, we can compare the observed distribution of beneficial alleles across multiple populations. The patterns of selective sweeps and adaptive fixations that are exclusive to or shared among multiple populations can be used to measure a beneficial allele's geographic extent, which is influenced by the factors outlined above. If we infer multiple structured populations have fixed the same beneficial allele, this suggests that pleiotropy has not disrupted the adaptive value of the allele across environments or that the populations share a sufficiently similar set of selective pressures. Assessment of the relative frequency and geographic extent of unique and shared beneficial alleles thus allows us to quantify the scale of local adaptation. Additionally, when multiple populations do share an adaptive allele, we can infer the mode by which sharing occurred (Lee and Coop 2017), providing further insights about the environmental and genetic context of each adaptation as well as the processes underlying allele sharing among populations.

Motivated to improve our understanding about the genetic basis of local adaptation and its geographic scale, we set out to use the reverse ecology approach to understand patterns of adaptation via selective sweeps in multiple discrete populations of domesticated maize Zea mays ssp. mays and its wild relative teosinte Zea mays ssp. parviglumis growing across their native range in Mexico. Zea mays is an annual grass, native to southern Mexico. Maize was domesticated $\approx 9,000$ years ago (Piperno et al. 2009) from its common ancestor with the extant annual grass teosinte, but traditional open-pollinated populations maintain an extremely large population size and a surprising amount of diversity (Bellon et al. 2018). Maize is also the world's most productive crop (Ranum et al. 2014), and an important model system for genetics (Nannas and Dawe 2015).

Previous work in both maize and teosinte has demonstrated clear population structure at both regional (Pyhäjärvi et al. 2013) and fine (Van Heerwaarden et al. 2010) scales, and population genetic and common garden studies in both subspecies have shown clear signatures of populations being adapted to their ${ }^{163}$ ecological conditions. In maize this includes local adaptation to 164 high elevation (Fustier et al. 2019; Gates et al. 2019), phosphorous 165 (Rodriguez-Zapata et al. 2021), temperature (Butler and Huybers 166 2013), and day length (Swarts et al. 2017). Similarly, studies of ${ }_{167}$ teosinte have documented local adaptation based on features 168 such as the differential patterns of microbial community recruitment (O'Brien et al. 2019), elevation (Fustier et al. 2019, 2017), and temperature and phosphorous (Aguirre-Liguori et al. 2019).

Studying local adaptation of maize and teosinte across the same geographic locations presents opportunities to disentangle multiple processes that interact with adaptation. For example, the effect of the domestication process in maize populations and their ongoing interaction and dependence on humans has created changes in the timing and types of selection imposed across all populations, as well as changes in demography (Wright et al. 2005). Based on population structure and differences in the abiotic environment among populations, we anticipated that local adaptation would have a small geographic scale. We predicted that sweeps would be exclusive to individual populations, and that adaptations shared between subspecies would be limited to sympatric pairs of populations growing in similar environments and with ample opportunity for genetic exchange. Because of domestication and the ongoing migration facilitated by humans, we expected that maize would show more shared adaptations, leading to a relatively larger geographic scale. Contrary to our predictions, our results suggest adaptations are often shared across two or more populations, and are commonly between maize and teosinte, rather than being exclusive to individual populations. We also found that migration and standing variation have played an important role as a source of beneficial alleles, including many that are shared across the two subspecies.

\section{Results}

We sampled teosinte (Zea mays subsp. parviglumis) individuals from six locations across its native range, along with a nearby (sympatric) population of traditionally cultivated openpollinated maize (commonly referred to as landraces) at five of these locations (Figure 1C). We sampled ten individuals from each population for each subspecies, with the exception of the Palmar Chico populations, where we took advantage of 55 and 50 individuals previously sampled for maize and teosinte, respectively (Table S1, Supplement I, Chen et al. 2020). In most cases, results for both Palmar Chico populations were downsampled to ten randomly selected individuals to facilitate comparisons to the other populations. However, we used a second random sample to assess false positives in our inference of selective sweeps (see Results and Methods)

To further evaluate patterns of local versus geographically widespread adaptation, we compared many results of our geographically well-defined populations to results found over the entire study range (referred to as "rangewide" throughout). Rangewide samples for each subspecies were constructed by randomly selecting one individual from each population. All 195 individuals were sequenced at 20 to $25 x$ coverage and aligned to version 5 of the Zea mays B73 reference genome (Hufford et al. 2021; Portwood et al. 2019). Analyses were based on genotype likelihoods (Korneliussen et al. 2014) except in cases where called genotypes were required (see Methods).

\section{Subspecies and populations are genetically distinct despite evidence of gene flow.}

To assess the relationships among our sampled populations, we constructed a population-level phylogeny using treemix (Pickrell and Pritchard 2012 v.1.13). As anticipated from previous work (Buckler and Holtsford 1996; Hufford et al. 2012), we found clear divergence between two clades composed of maize and 
teosinte populations, though the relationship among geographic locations differed between the subspecies clades (Figure 1B).

Within subspecies, populations were genetically distinct from one another. Using NGSadmix (Skotte et al. 2013), there was little evidence of admixture between populations of the same subspecies; only two of the sampled individuals revealed mixed population ancestry (Figure 1A and 1D).

Despite the clear phylogenetic separation between the two subspecies, there is evidence for gene flow between maize and teosinte populations. We conducted f4 tests using treemix (Pickrell and Pritchard 2012), and found that all populations showed some evidence of gene flow with various populations of the other subspecies, as measured by the high absolute Z-Scores of the $\mathrm{f} 4$ statistic. However, Z-Scores were sensitive to the specific combinations of non-focal populations included in each test (Table S2). More specifically, we found that elevated f4 tests almost always included the maize population from Crucero Lagunitas $\left(p<2 \times 10^{-10}\right)$, which was true whether or not the $\mathrm{f} 4$ test included its sympatric teosinte, and more generally, there is little evidence for increased geneflow between sympatric pairs (Figure 1E).

\section{Populations vary in their diversity, demography, and history of inbreeding}

We estimated pairwise nucleotide diversity $(\pi)$ and Tajima's $\mathrm{D}$ in non-overlapping $100 \mathrm{~Kb}$ windows along the genome in our sampled populations using ANGSD (Korneliussen et al. 2014). For all populations, $\pi$ was in the range of 0.006 to 0.01 , consistent with both previous Sanger (Wright et al. 2005) and short-read (Hufford et al. 2012) estimates for both subspecies. Variation in Tajima's D and $\pi$ was greater among populations of teosinte than maize (Figure 2D, Table S2).

We independently estimated the demographic history for each population from their respective site frequency spectra using mushi (DeWitt et al. 2021 v0.2.0). All histories estimated a bottleneck that started approximately 10 thousand generations ago (assuming a mutation rate of $3 \times 10^{-8}$ (Clark et al. 2005) (Figure 2E).

Teosinte is a primarily outcrossing grass (Hufford 2010), and regional maize farming practices promote outcrossing as well (Bellon et al. 2018). To validate our estimated demography and characterize the history of inbreeding in each population, we compared the empirical quantiles of homozygosity by descent (HBD) segments inferred using IBDseq (Browning and Browning 2013) to those simulated under the demography of each population. With the exception of the smallest HBD segments, which are more prone to inaccurate estimation, the simulated quantiles generally resemble the empirical quantiles (Figure 2D). This indicates that the inbreeding history of our population is adequately captured by the demography. However, consistent with previous studies of teosinte (Hufford 2010), we do see ${ }^{23}$ variation in the distributions of HDB among populations. For 238 example, the size distribution of HBD segments in San Lorenzo 239 and Los Guajes were consistently larger than those simulated 240 from their demographies, particularly for the smallest segments. ${ }^{241}$ This likely reflects inbreeding caused by demographic changes, 242 particularly those further in the past that were not detected in 243 our demography inferences. These results are consistent with ${ }_{244}$ previous studies, that found evidence for historical inbreeding in 245
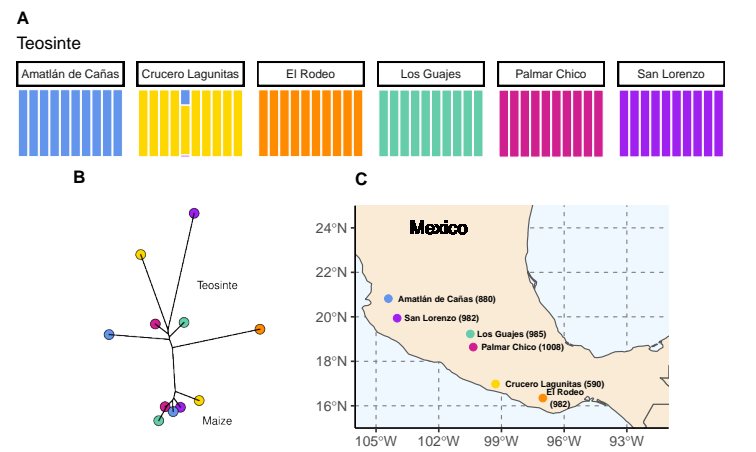

D

Maize
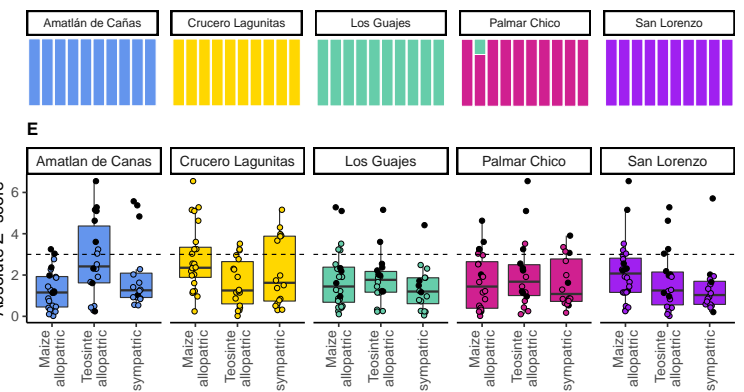

Figure 1 | The geographic distribution, population structure, and gene flow of maize and teosinte populations. (A and D) Admixture proportions among populations within subspecies. The dominant cluster in each population is colored by sampling location. (B) The unrooted tree of maize and teosinte populations. (C) Geographic sampling locations for the studied maize and teosinte populations. (E) F4 tests to quantify evidence of gene flow between the subspecies for allopatric and sympatric population pairs. Each point in (E) reports the absolute Z-Score for an $\mathrm{f} 4$ test, where a given focal population was partnered in a phylogenetic tree with another population of the same subspecies as a sister node, and two other populations from the other subspecies as a sister clade (see Methods for further details). Black points show $\mathrm{f} 4$ tests that included maize from Crucero Lagunitas, otherwise points are colored by focal population. Dotted line corresponds to our chosen significance threshold $(p=0.001)$.

teosinte, particularly in individuals sampled from San Lorenzo (Pyhäjärvi et al. 2013). Lastly, we estimated average pairwise inbreeding coefficients (F) using ngsRelate (Hanghøj et al. 2019). Although for some pairs of individuals $\mathrm{F}$ was as high as 0.37 , the mean value and $\mathrm{F}$ was $0.017 \pm 0.001$ (SE) and $0.033 \pm 0.001$ (SE) for maize and teosinte (respectively), (Figure 2E), suggesting there has been relatively little inbreeding in either subspecies in the recent past.

\section{Linked selection has a larger effect on diversity in maize}

Census size is known to vary widely among maize and teosinte populations (Hufford 2010; Wilkes et al. 1967), yet genomewide pairwise sequence diversity $(\pi)$ was relatively similar across our studied populations (Figure 2). Under strictly neutral models, census size differences should translate into differences in genetic diversity. Widespread selection at linked sites, however, can attenuate correlations between census size and diversity (Lewontin et al. 1974; Corbett-Detig et al. 2015; Buffalo 2021), and 

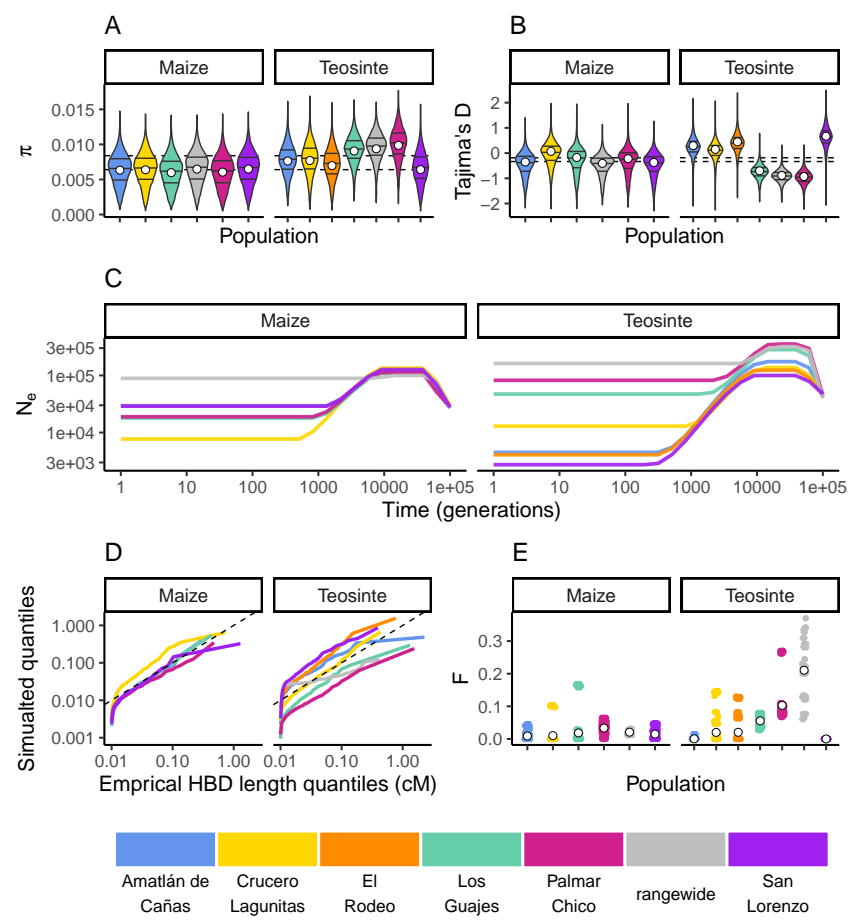

Figure 2 I Inbreeding, diversity, and demography. The distribution of $\pi(\mathrm{A})$ and Tajima's D (B) calculated in $100 \mathrm{~Kb}$ windows for maize and teosinte populations. Dashed lines show the median values for the two subspecies. Filled white points show the median values of each statistic generated from coalescent simulations under the demographic history inferred for each population. Colors for each population are as in Figure 1 and are shown at the bottom of the figure. (C) The inferred demography for each population. (D) The quantile of observed Homozygosity By Descent (HBD) lengths (cM) versus those simulated under each population demography. Dashed lines shows the 1:1 correspondence between the axes. (E) The distribution of inbreeding coefficients among pairs of individuals in each population. Filled white points are the average values for each population. previous work has demonstrated differential impacts of linked selection on diversity in maize and teosinte (Beissinger et al. 2016).

In an effort to better understand the forces shaping varia- ${ }^{310}$ tion among our studied populations, and to be able to better ${ }^{311}$ assess the reliability of our demographic inferences (which ig- ${ }^{312}$ nored linked selection), we fit a model of linked selection to ${ }^{313}$ predict $\pi$ from estimates of recombination rate and the density ${ }^{314}$ of functional base pairs. As recombination rate increases, neutral 315 variants are able to disassociate from nearby selected variants. ${ }^{316}$ Combined with gene annotation information, this signature al- 317 lowed us to estimate what the population scaled mutation rate ${ }^{318}$ $\left(\theta=4 N_{e} \mu\right)$ would be in the absence of linked selection (Coop ${ }^{319}$ and Ralph 2012; Corbett-Detig et al. 2015).

We used AIC (Wagenmakers and Farrell 2004) to select between different models that predicted neutral $\theta$, finding that the ${ }^{321}$ full linked selection model, which includes background selection ${ }^{322}$ and hitchhiking parameters, was favored for all populations. In ${ }_{323}$ all populations, the estimated confidence intervals of neutral
$265 \theta$ from the full model were non-overlapping with that of the 266 intercept-only model which provides an estimate of the aver267 age genomewide value of $\pi$ (Figure 3B). The two estimates of ${ }_{268} \theta$ are relatively similar for all teosinte populations, while the 269 difference was consistently larger in maize, suggesting linked 270 selection has played a more important role in shaping maize diversity (Figure 3B). Similarly, the expected value of $\pi$ approaches the estimated neutral diversity at lower recombination rate in teosinte (Figure 3A). Specifically, averaged across populations, $\pi$ in teosinte reached $95 \%$ of the neutral $\theta$ estimate at a minimum recombination rate of $0.01 \mathrm{cM} / \mathrm{Mb} \pm 0.0006$ (SE) compared to $1.04 \mathrm{cM} / \mathrm{Mb} \pm 0.05$ (SE) in maize. Relatedly, in maize, approximately $92 \% \pm 0.2 \%$ (SE) of the available genomic windows fell below the recombination rate minimum described above, compared to $12 \% \pm 0.9 \%$ in teosinte. Previous inferences of linked selection in maize and teosinte found the reverse pattern, with a strong impact of linked selection in teosinte (Beissinger et al. 2016). One potential explanation for this discrepancy is that previous analyses did not directly estimate $\theta$, but instead assumed that levels of $\pi$ distant from coding sequences were reflective of the true neutral diversity. This approach would underestimate the effects of linked selection we observed in maize, where the majority of the genome has levels of $\pi$ below $\theta$. Overall, our results indicate that linked selection has played a larger role in shaping diversity for maize than teosinte populations.
290

291

07

9$$
\text { . }
$$
(1) 2

b tytion type (see Figure S3, Supplement IV), the patterns are the opposite of that found in Arabidopsis, perhaps because of the increased level of methylation in maize and the higher mutation rate at methylated cytosines. Even after accounting for differences among mutation types, rangewide values remained commensurate with that of the populations.

\section{The proportion of mutations fixed by natural selection $(\alpha)$ is associated with neutral diversity in teosinte}

The variable demographic histories of our populations may have affected the process of adaptive evolution. Under a neutral mode 

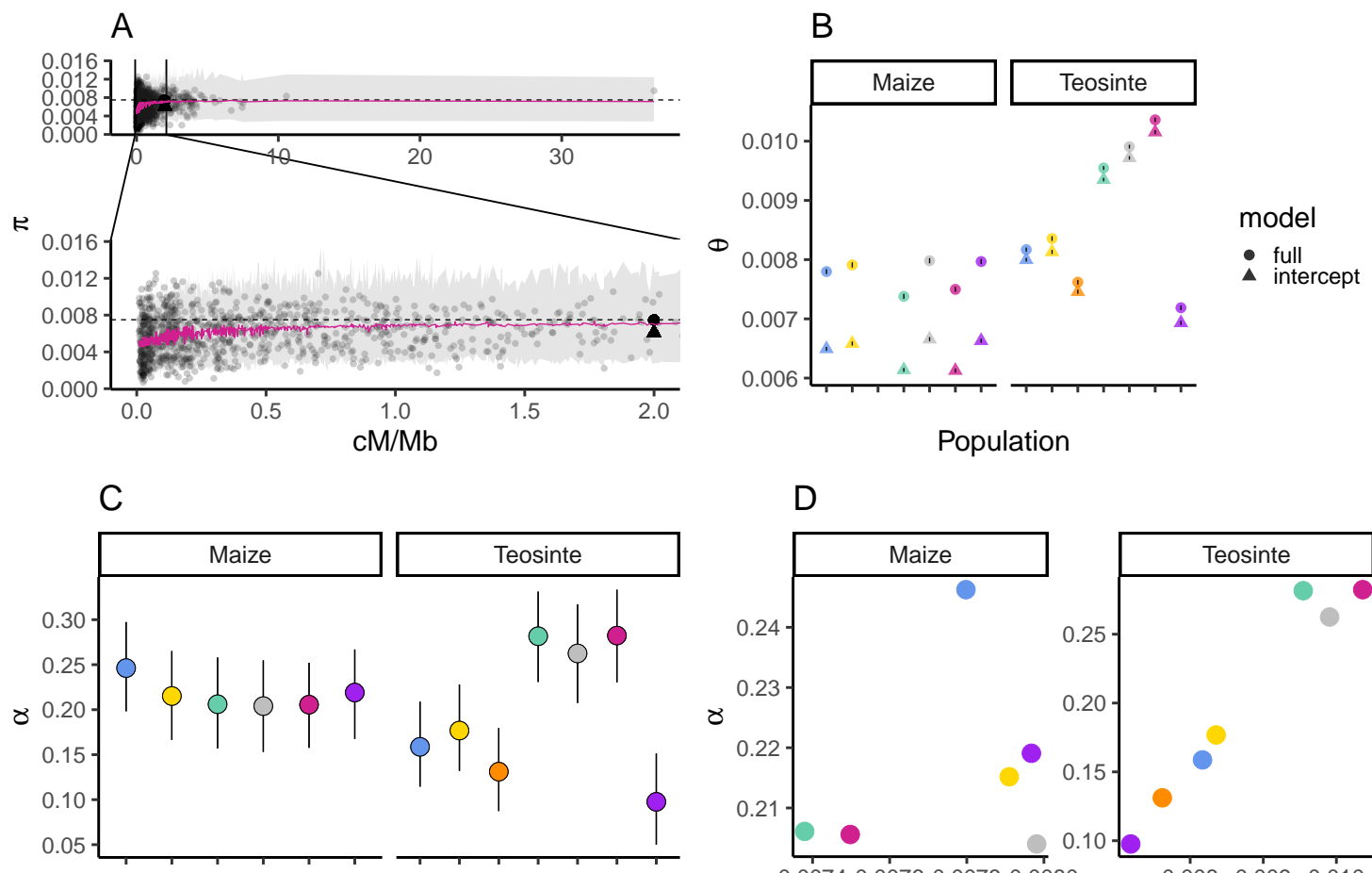

Population
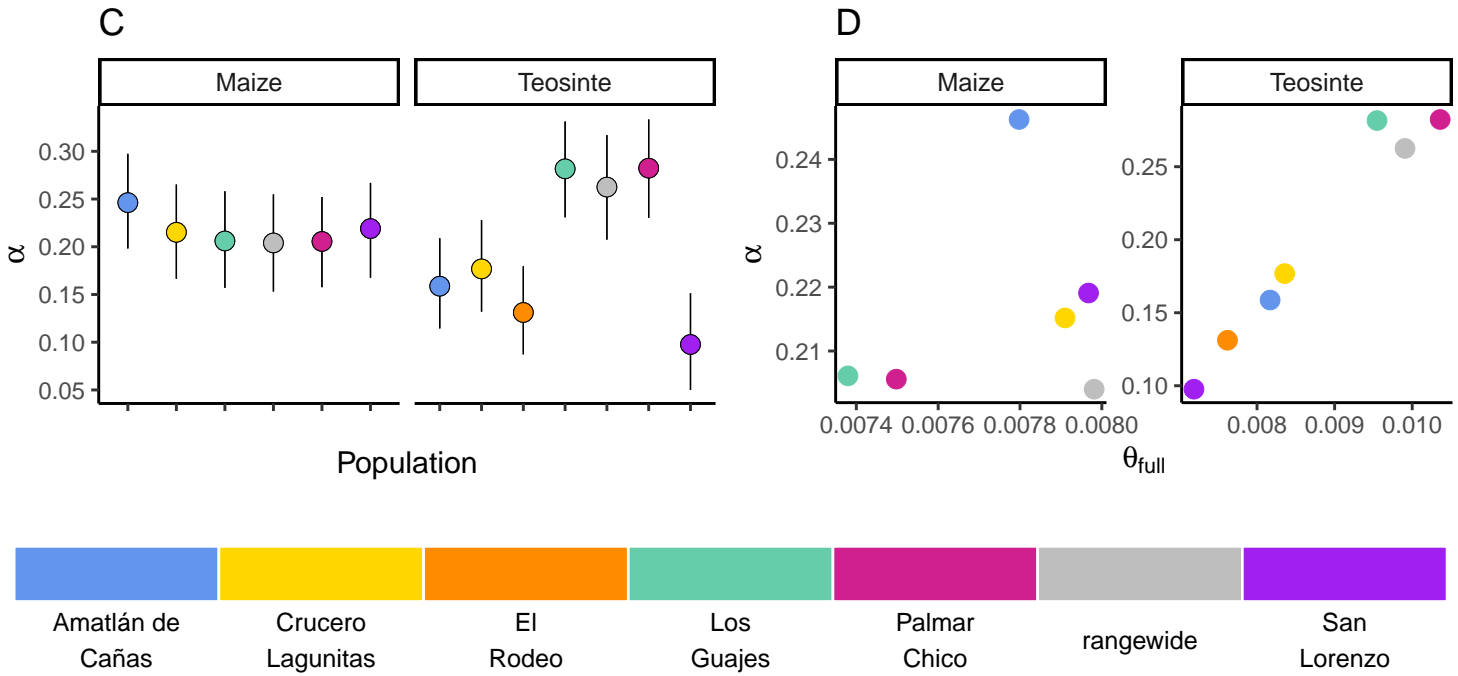

Figure 3 Linked selection and the proportion of mutations fixed by natural selection. (A) The relationship between recombination rate $(\mathrm{cM} / \mathrm{Mb})$ and diversity $(\pi)$ measured in $10 \mathrm{~Kb}$ windows, overlaid with the full linked selection model fit (in purple; data and fit shown for maize from Palmar Chico only). Grey bands show the 95\% prediction interval of the full model fit. The dotted line shows the maximum likelihood estimate of neutral $\theta$. Bottom of (A) shows zoomed in region of higher density. The circle and triangle at the edge of the zoomed in plot show the maximum likelihood estimates of $\theta$ for the full and intercept models (see Methods), as do the points shown in panel B. (B) The maximum likelihood estimates of $\theta$ for the full and intercept models for each population, respectively. (C) Estimated values of the proportion of mutations fixed by natural selection $(\alpha)$. Vertical lines show the $95 \%$ credible interval. (D) The relationship between $(\alpha)$ and the maximum likelihood estimate of $\theta$ from the full linked selection model for each population.

of evolution, population size and selection have no impact on ${ }_{342}$ the rate of substitutions, which are predicted to be equal to the ${ }_{343}$ mutation rate. When adaptive mutations are introduced, theory predicts that the rate of substitutions depends on the mutation rate, effective population size, and the strength of selection (Gillespie 2004). It follows that populations with larger longterm effective populations sizes should have a higher proportion 346 of substitutions that are adaptive.

Motivated by theory, we used simulations to confirm that $\alpha{ }_{349}$ increases with population size, and that the relationship is robust 350 to variation in recent demographic changes and the distribution 351 of fitness effects (table S3 and figure S2). We then investigated 352 the empirical relationship between $\theta$ and $\alpha$ in our populations ${ }_{353}$ by testing for an association between the two separately for each 354 subspecies. We used the mean $\alpha$ predictions and associated 355 them with the values of neutral $\theta$ estimated from the full linked 356 selection model. We found that $\theta$ and $\alpha$ are strongly correlated 357 for teosinte populations $\left(r=0.97, p<3.6 \times 10^{-9}\right)$, but not for maize $(r=0.09, p=0.77)$ (Figure 3).

\section{Teosinte populations have a higher proportion of private} sweeps

Our inferences of $\alpha$ are based on substitutions at nonsynonymous sites. The functional space for selection to act on occurs over many other parts of the genome besides protein coding bases, especially for large repetitive plant genomes (Mei et al. 2018). To identify signatures of adaptation occurring anywhere in the genome, we used RAiSD (Alachiotis and Pavlidis 2018) to identify putative selective sweeps in each population, where sweeps were selected using an outlier cutoff defined by the $99.9 \%$ percentile of the $\mu$ summary statistic for data generated using coalescent simulations under each population's estimated demography (see Methods). Simulations suggest this approach has high accuracy and power compared to alternative 

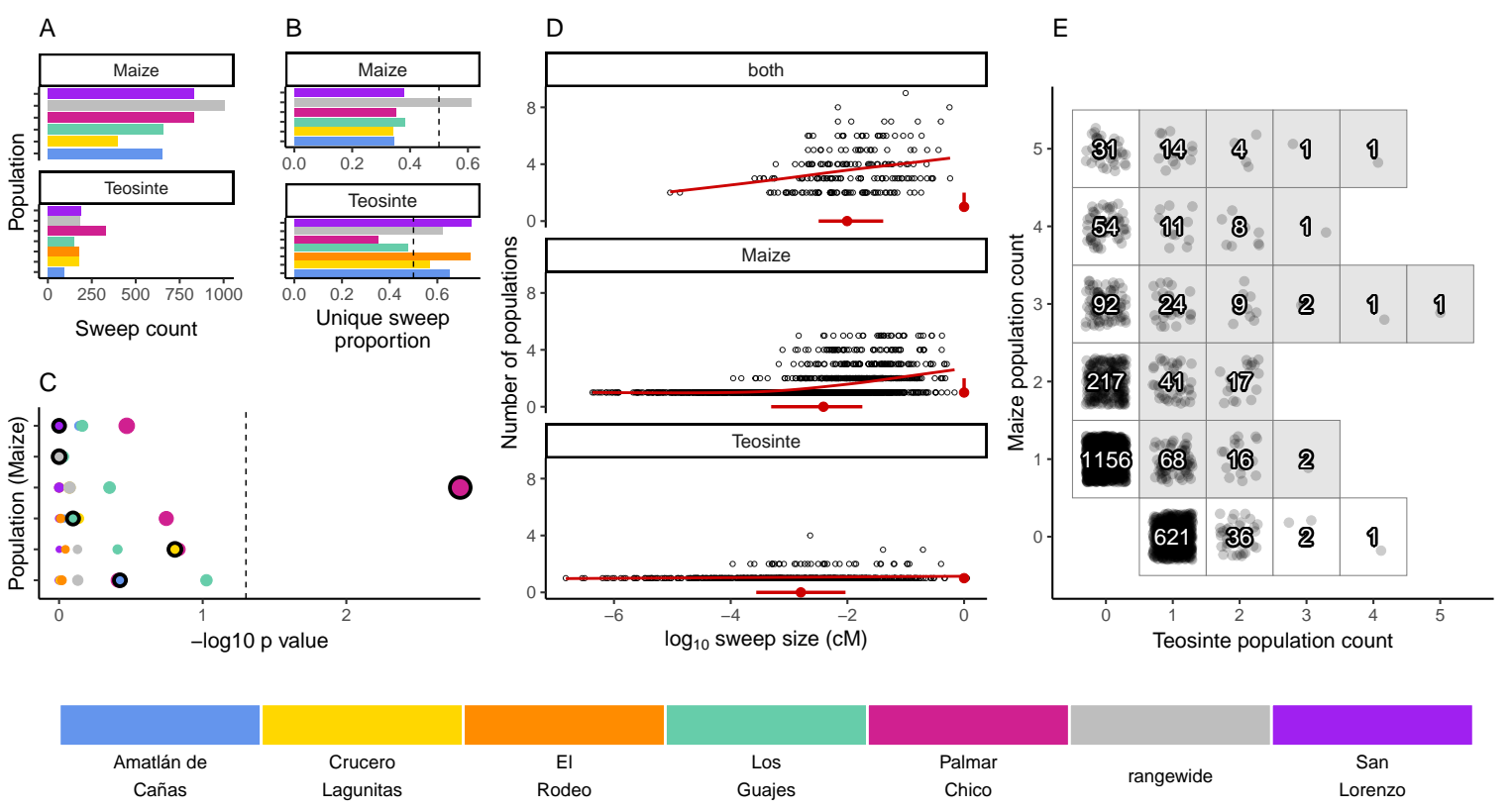

Figure 4 The distribution of shared and private selective sweeps. (A) The total number of sweeps inferred in each population. (B) The proportion of sweeps that are unique to each population. Dotted line drawn at $50 \%$. (C) The negative $\log 10 \mathrm{p}$ values for hypergeometric tests to identify maize-teosinte population pairs that shared more sweeps than expected by chance (see Methods). P values were adjusted for multiple tests using the Benjamini and Yekutieli method. Populations along the y axis are maize (order matches the legend below, with Amatlán de Cañas on bottom), while the point color designates the teosinte population each maize population was paired with. Points with black outlined highlight the sympatric population comparisons. Point size is scaled by the number of shared sweeps identified in each pair. The dotted line indicates our chosen significance level $(p=0.05)$. (D) The relationship between genetic map length of sweep regions and the number of populations each sweep region occurred in, split for sweeps shared in two or more populations of both subspecies, exclusive to maize, or exclusive to teosinte. The black line shows a loess smooth fit. Grey points and bars at the margin of each subplot show the 50th, 25th and 75th percentiles of sweep sizes, respectively. (E) Counts of shared and unique sweeps broken down by how many maize and teosinte populations they occurred in. Grey boxes show sweeps shared across the two subspecies.

methods over a broad range of demographic and sweep scenar- 383 ios (Alachiotis and Pavlidis 2018). To further assess the accuracy 384 of our sweep inferences, we compared the overlap in sweep 385 regions from a second random sample from both maize and 386 teosinte from Palmar Chico. We estimated a false positive rate 387 to be 0.6 and 0.5 for maize and teosinte, respectively, suggesting 388 many of the putative sweep regions in other populations are also false positives, and that the total number of sweeps we have identified is likely an overestimate. Approximately $10 \%$ of all base pairs fell within a sweep region found in one or more of the sampled populations, although the true proportion is likely lower based on our estimated false positive rate. The density of coding sequence was lower in sweep regions (0.029 coding sequence base pairs/sweep base pairs) than the genome-wide gene density ( 0.042 coding sequence base pairs/total base pairs).

We used the inferred sweep regions to assess the degree to 397 which adaptation is shared or locally restricted using the sweep 398 regions we identified. We determined how many sweep regions 399 were exclusive to one population (private), along with the num- 400 ber and size of overlapping sweep regions shared across two 401 or more populations within and between the two subspecies. 402 Overall, sharing was common, though fewer sweeps were ex- 403 clusively shared between teosinte populations (Figure 4A). In 404 teosinte populations, $59 \%$ of sweeps were private, which was 405 significantly greater than the $40 \%$ found in maize (binomial glm, 406 $p=2.63 \times 10^{-7}$; Figure 4B). We saw a general pattern that larger sweep regions tended to be shared between more populations, though the pattern was negligible for sweeps exclusive to teosinte (Figure 4D). Additionally, 31 out of 1550 sweep regions were exclusive to and shared by all maize populations, compared to 0 out of 660 for teosinte.

\section{Sympatric population pairs do not share more sweeps}

If local adaptation favors certain alleles in a given environment, we might expect to see increased sharing of sweeps between sympatric populations of maize and teosinte. To look for evidence of such higher sharing, we used a hypergeometric test based on the number of sweeps in each population, and the number of shared sweeps between population pairs, which allowed us to test if sympatric population pairs tended to have more sharing than expected by chance. In conducting this test, we incorporated the estimated false positive rate (see Methods). Sympatric pairs did not tend to have a lower $\mathrm{p}$ value than allopatric pairs, and only the population pair in Palmar Chico showed more sharing than expected by chance (Figure 4). We additionally found that, despite evidence that sweeps are commonly shared between maize and teosinte (Figure 4C), there were zero sweeps exclusive to sympatric pairs; sweeps that were shared between sympatric pairs always included at least one other allopatric population. 


\section{Convergent adaptation from migration is common among ${ }_{467}$} maize and teosinte populations

In instances when two or more populations shared a sweep re- ${ }^{469}$ gion, we used rdmc (Lee and Coop 2017; Tittes 2020) to infer the ${ }^{470}$ most likely mode of convergence. We classified sweeps based ${ }^{471}$ on which composite log-likelihood model was greatest out of 472 four possible models of convergence (independent mutations, ${ }^{473}$ migration, neutral, and standing variation). Of the 1734 sweeps ${ }^{474}$ that were shared by two or more populations, there were $17,663,475$ 627 , and 427 sweeps inferred to be convergent via independent 476 mutations, migration, neutral, and standing variation, respec- ${ }^{477}$ tively (Figure 5C). While the high proportion of neutral models 478 inferred by rdmc is consistent with a relatively high false posi- ${ }^{479}$ tive rate, confirmation of non-neutral patterns of diversity from ${ }^{480}$ this second approach increases our confidence that regions iden- ${ }^{48}$ tified for other modes indeed represent sweeps. The strength ${ }^{48}$ of support (measured as the composite likelihood score of the ${ }^{483}$ best model relative to the next best) varied among sweeps and ${ }^{484}$ modes of convergence, but in general a single model tended to be clearly favored among the alternatives (Figure 5A). Selection coefficients for sweeps varied among modes, with convergence ${ }^{487}$ via migration having the highest average estimate (Figure 5B). When migration was the mode of convergence and sweeps were shared by both subspecies, teosinte Palmar Chico and maize from Crucero Lagunitas were the most frequent source populations (Figure 5D). In convergence models with migration, we considered only a low and high migration rate, set to 0.001 and 0.1 , respectively. The most likely migration rate varied across sweeps, but the migration rate was high for a majority of sweeps shared by the two subspecies and sweeps exclusive to maize. In contrast, the low migration rate was always most likely for sweeps exclusive to teosinte (Figure 5F). Together, these findings indicate that many alleles are adaptive in the genomic background of both maize and teosinte, and that adaptive alleles are commonly shared between the two subspecies.

\section{Discussion}

\section{Local adaptation occurs at intermediate scales}

Our findings overall suggest that adaptation in maize and teosinte does occur locally, but frequently at scales larger than individual populations. Gossmann et al. (2010) hypothesized that population structure within a species could limit the fixation of adaptive alleles across a species range, causing a reduction in the proportion of mutations fixed by positive selection $(\alpha)$. Based on this hypothesis and the strong population structure we observed (Figure 1), we expected that rangewide samples would have a smaller estimates of $\alpha$. Instead, $\alpha$ for the rangewide samples of both maize and teosinte were commensurate with that of individual populations (Figure 3), a pattern that persisted even when we considered $\alpha$ estimated from several different mutation types (Figure S3). This is inconsistent with the patterns we would expect fine-scale local adaptation to generate, where adaptive substitutions for a given population should not ${ }_{520}$ be shared by other populations experiencing their own distinct local selective pressures.

We found a similar pattern from our analysis of shared ver- ${ }_{523}$ sus unique selective sweeps, which were more often shared by 524 at least one other allopatric population. Similar to our predic- 525 tions for $\alpha$, we expected that local adaptation would lead most 526 sweeps to be exclusive to individual populations. Instead, the 527 average proportion of sweeps exclusive to a single population 528 was low to moderate for maize and teosinte populations, respectively (Figure 4). We also expected that maize and teosinte populations growing in close proximity would share similar local selective pressures and would therefore share more signatures of adaptation. However, only maize and teosinte sampled from Palmar Chico showed evidence of sharing more sweeps than would be expected by chance, and overall sympatric pairs did not show increased sharing of selective sweep regions compared to allopatric pairs (Figure 4). The regional scale of local adaptation is consistent with patterns seen in maize adaptation to the highlands (Calfee et al. 2021), where sympatric maize and teosinte populations show little evidence of adaptive gene flow, and adaptive teosinte introgression appears widespread among highland maize. To our surprise, private sweeps made up a higher proportion of sweeps for the rangewide sample of maize. One possible explanation is that rangewide samples are enriched for older sweeps that are shared across all populations but difficult to detect in any single sample. Consistent with this, we find evidence that the sweep statistic $\mu$ for individual maize populations was elevated in the sweep regions exclusive to the rangewide sample, but did not reach the cutoff to be considered outliers (Figure S6, 22).

There are a number of considerations to make in the interpretation of our results. The two methods we used to identify signatures of adaptation, estimating $\alpha$ and identifying signatures of selective sweeps, both depend on the fixation of beneficial mutations. For the moderate population sizes and selection coefficients observed here, fixation of new beneficial mutations takes a considerable amount of time, on the order of $4 \log (2 N) / \mathrm{s}$ generations (Charlesworth 2020) of thousands of years. Compared to the sojourn time of adaptive mutations, our populations may have occupied their current locations for relatively few generations. As a result, the selective sweeps underlying local adaptation to the selective pressures that populations currently face are more likely to be incomplete, so may be more difficult to detect (Xue et al. 2021; Pritchard et al. 2010). Likewise, the adaptive sweeps that have completed - and are more likely to be detected - may have been under selection in ancestral populations that occupied different environments than that of the sampled individuals. Another complication in detecting local adaptation relates to the size and complexity of plant genomes. Large genomes may lead to more soft sweeps, where no single mutation driving adaptive evolution would fix (Mei et al. 2018). Like incomplete sweeps, soft sweeps are harder to identify (Schrider and Kern 2016; Pritchard et al. 2010), which could obscure the signatures of local adaptation. Even if our populations have occupied their current locations for a sufficient duration for local adaptation to occur, the completion of selective sweeps may be hindered by changes and fluctuations in the local biotic and abiotic conditions. Relatively rapid change in local conditions could result in fluctuating selection, such that most alleles do not remain beneficial for long enough to become fixed (Rudman et al. 2021).

While our focus has been on the trajectory of individual beneficial alleles, the genetivc basis of many adaptive traits may be highly polygenic. Allele frequency changes underlying polygenic adaptation are more subtle than those assumed under selective sweeps, making them harder to detect (Pritchard et al. 2010). Evaluating local adaptation in maize and other systems will be facilitated by studying the contribution of polygenic adaptation to the evolution of complex traits. However, if adaptation across our studied populations were strictly polygenic, 


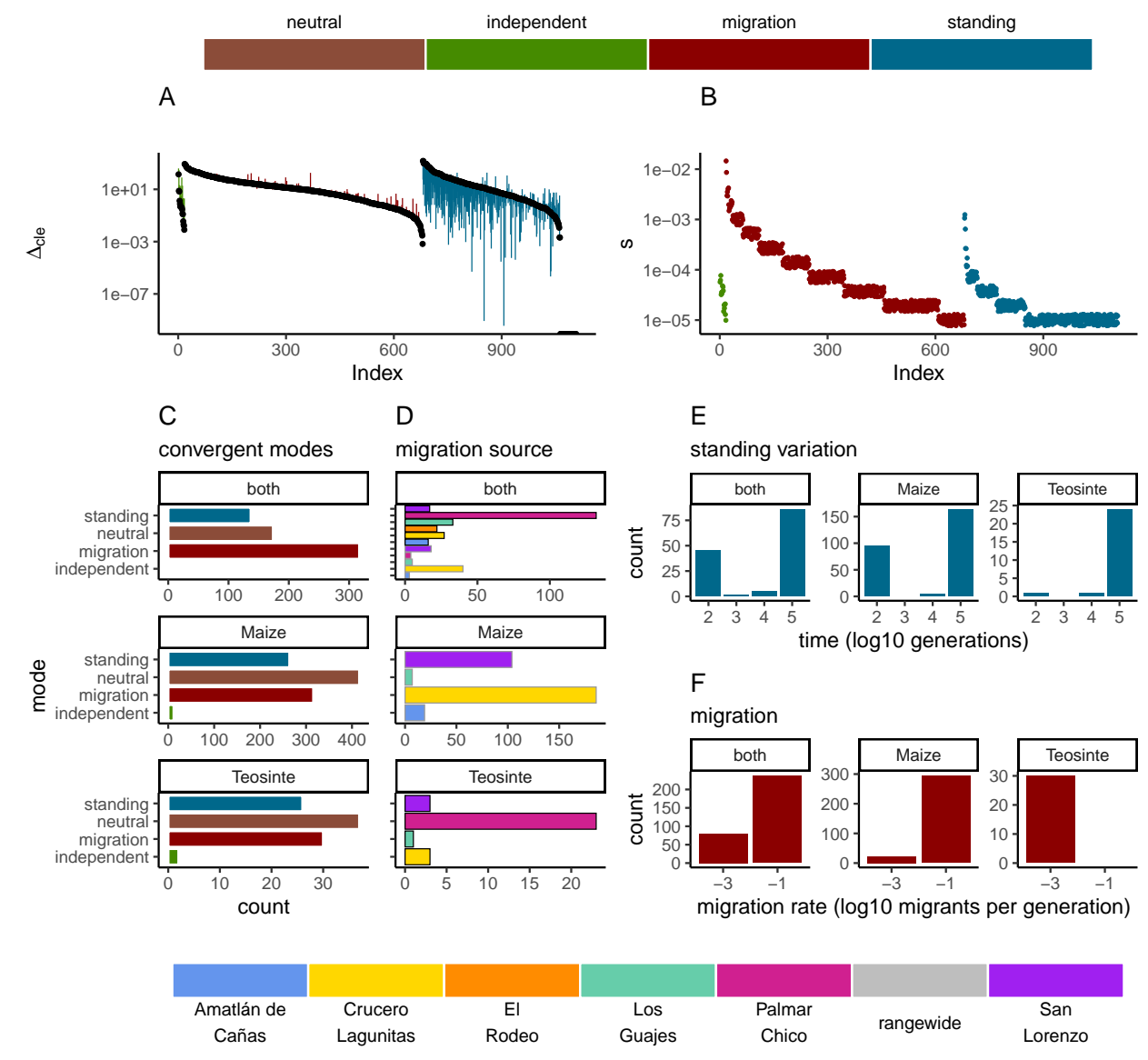

Figure 5 Modes of convergent adaptation and affiliated parameters for shared selective sweeps. (A) The difference in composite likelihood scores for the best supported mode of convergent adaptation (colors in top legend) compared to next best mode (black points), and best mode compared to the neutral model (other end of each line segment above or below black point). (B) Selection coefficients colored by the most likely mode of convergent adaptation. (C) Number of shared sweeps for both subspecies that were inferred to be from each convergent adaptation mode. (D) The most likely source population for shared sweeps that converged via migration. Bars are colored by population (bottom legend) and are outlined in black for teosinte and grey for maize. (E) Observed frequency of the inferred time in generations that each selected allele persisted prior to selection for models of convergent adaptation via standing variation. (F) Observed frequency of each inferred migration rate value for models of convergent adaptation via migration. Panels C, D, E and F are partitioned by which subspecies shared the sweep.

and especially if it were acting on alleles with small effect sizes, 545 we would expect to find little or no shared sweeps. The fact that ${ }_{546}$ we find many instances of sharing across populations supports ${ }_{547}$ that a non-trival amount of local adaptation is occurring via 548 selective sweeps, or through polygenic adaptation acting on a 549 few loci with large effects that leave a signature similar to that 550 of a selective sweep.

\section{Differences in diversity and demography influence adaptation in maize and teosinte}

While our results were generally similar between the two subspecies and among the sampled populations, there are several 556 important differences. The most obvious difference between 557 the subspecies is the ongoing interaction and dependence of 558 maize on humans via domestication and farming. Compared 559 to teosinte, maize had lower average genomewide estimates 560 of diversity (Figure 2A), which persisted after accounting for 561 the effects of linked selection (Figure 3B). These differences are consistent with the previously discovered pattern that diversity tends to be lower in crops compared to their wild relatives (Doebley 1989; Hufford et al. 2012), a pattern putatively driven by domestication bottlenecks (Eyre-Walker et al. 1998). In line with this argument, the few teosinte populations with lower diversity than those in maize (El Rodeo and San Lorenzo) were inferred to have the most substantial bottlenecks and historical inbreeding (Figure 2). More generally, we found that $\pi$ and Tajima's D were more variable among teosinte populations, indicative of differences in their demographic histories.

Our demographic inferences suggest that all populations had signatures of a bottleneck, the timing of which coincides with the beginning of maize domestication $\approx 9,000$ years ago (Piperno et al. 2009). The severity of the bottleneck varied considerably across populations, particularly for teosinte. While finding a bottleneck in the maize populations is consistent with domes- 
tication, it is less clear why we found a consistent bottleneck 624 for the teosinte populations at approximately the same time. 625 One possibility is that the teosinte bottlenecks reflect land use ${ }_{626}$ change induced by human colonization. For example, evidence ${ }_{227}$ from Mesoamerican phytolith records in lake sediment show ${ }_{228}$ evidence of anthropogenic burning as early as $11 \mathrm{~K}$ years B.P. ${ }_{629}$ (Piperno 1991). The establishment and spread of human popula- 630 tions over the subsequent millenia would require ever increasing 631 area for farming, dwellings, transportation, and trade (Haines 632 et al. 2000). Such land use changes would likely encroach on 633 the habitat available for teosinte and drive species-wide cen- ${ }_{634}$ sus size declines. Given the success of maize breeding and 635 domestication, we anticipated a recent expansion for maize pop- 636 ulations as previously seen (Beissinger et al. 2016; Wang et al. 637 2017). However, with only 10 individuals per population, recent 638 expansions will be difficult to detect (Keinan and Clark 2012; 639 Coventry et al. 2010). The demography of the rangewide sam- 640 ples for both subspecies showed little evidence of the bottleneck ${ }_{641}$ inferred in individual populations, likely due to the reduced ${ }_{642}$ sampled size (5 and 6 individuals) for the rangewide data. We ${ }_{643}$ additionally used strong regularization penalties to avoid over- 644 fitting (see Methods), which limits the detection of rapid and ${ }_{645}$ dramatic changes in population size. The near-constant size ${ }_{646}$ of the rangewide samples and the lack of recent expansion in ${ }_{647}$ maize are both likely influenced by this modeling choice. Lastly, 648 given the strong effects of linked selection inferred in maize, our 649 demographic models may have underestimated their effective 650 population sizes. However, all populations independently con- ${ }_{651}$ verged to similar values in the oldest generation times, around 652 the time when we would expect the ancestral lineages would ${ }_{653}$ have coalesced (Figure 2C). This suggests any biases in the esti- ${ }_{654}$ mated population sizes that are specific to maize are occurring 655 in the more recent past. Similar to the arguments above, this 656 provides another reason that recent population sizes in Maize ${ }_{657} 60$ may be underestimated.

Theory and simulations, including those presented here, sup- 659 port that the rate of fixation of new beneficial mutations should 660 increase with the population size $\left(N_{e}\right)$ and consequently the ${ }^{661}$ population-scaled mutation rate $\left(\theta=4 N_{e} \mu\right)$ (Gillespie 2004, Fig- 662 ures 3D and Supplement III). However, previous research in ${ }^{663}$ plants found little evidence for positive estimates of $\alpha$, and an 664 apparent lack of correlation between inferred population size 665 and $\alpha$ for new non-synonymous mutations (Gossmann et al. 666 2010). The discrepancy between those results and our own, in 667 regards to the overall estimates of $\alpha$, are likely explained by the 668 use of different methods and the sampling design. Our simu- 669 lations suggest the relationship between $\alpha$ and $\theta$ is positive but 670 non-linear. The rate of increase for $\alpha$ declines at larger values 671 of $\theta$ (Figure S2) This suggests finding an association between $\theta$ 672 and $\alpha$ may be sensitive (in theory and practice) on the parameter 673 space under consideration.

We found a significant correlation between neutral $\theta$ and $\alpha$, but only for teosinte populations (Figure 3C). While we consider this finding preliminary based on the small number of populations in the sample, the positive association found in teosinte populations is consistent with our simulations and some theory cited above that suggest larger populations increase the rate of adaptive fixation. However, it has also been suggested that the relationship between effective population size $\left(N_{e}\right)$ and $\alpha$ is driven by increased efficiency of purifying selection to reduce ${ }^{682}$ the fixation of nearly-neutral substitutions, rather than an in- 683 crease in the fixation rate of beneficial mutations per se (Galtier 684
2016; Hämälä and Tiffin 2020). We cannot confidently determine whether the association is driven by the increased efficiency of larger populations to purge weakly deleterious mutations or to fix adaptive ones. However, these are not mutually exclusive explanations, and both may contribute to differing degrees in actual populations. The lack of correlation in maize is likely driven by the recent divergence between populations and the strong recent selection during domestication that is shared by all the populations. As a result, populations have not had sufficient time for differences in population size to impact patterns of adaptive fixation. As recent changes in environment and population size are likely important aspects of the biology of many domesticates, we predict that the observed lack of correlation between $\theta$ and $\alpha$ in maize may be a common pattern across many taxa. However, another important caveat concerning these associations is the lack of independence among lineages, particularly for maize populations, which have diverged more recently. Further, studies with larger sample of populations may correct for non-independence when testing the associations between $\alpha$ and $\theta$, which would bolster the validity of any associations (or lack there) found.

Although the asymptotic MK method we employed has been shown to provide reliable estimates of $\alpha$ when fixations are due to strong beneficial mutations (Messer and Petrov 2013), it does not account for the influence of background selection and the rate of fixation of weakly beneficial mutations (Uricchio et al. 2019). Our analyses suggests linked selection is acting similarly across populations within subspecies (Figure 3B). As such, while we may be systematically underestimating $\alpha$, the bias is unlikely to change our general conclusions.

Another consideration is that our estimates of $\alpha$ contrasted non-synonymous and synonymous sites only. We observed a lower density of coding sequence in sweep regions, suggesting selective sweeps are more often acting on non-coding regulatory regions rather than modifying protein coding sequences directly. This result is consistent with the proposed importance of noncoding sequences for adaptation in species with large genomes (Mei et al. 2018) and highlights the importance of studying signatures of adaptation using multiple methods and regions of the genome in order to construct a comprehensive history.

Differences in adaptation between maize and teosinte, and among populations, were apparent based on differences in the patterns of selective sweeps. Maize had a higher proportion of selective sweeps shared with at least one other population (Figure S1). The greater number of shared sweeps in maize populations is likely the result of their recent shared selective history during the process of domestication, resulting in a set of phenotypes common to all maize (Stitzer and Ross-Ibarra 2018). In comparison, the higher proportion of unique sweeps in teosinte suggests local adaptation has played more of a role in shaping their recent evolutionary history. Teosinte grows untended, and did not undergo domestication, leaving more opportunity for divergence and local selection pressures to accumulate differences among populations. This is reflected in the inferred population history, which had longer terminal branch lengths for teosinte (Figure 1B), suggesting there is increased genetic isolation among teosinte populations due to longer divergence times, reduced gene flow, or both.

\section{Convergent adaptation is ubiquitous}

We found convergent adaptation to be common among populations and subspecies (Figures 4 and 5). The frequency of 
convergence further suggests there are a large number of mu- 747 tations that are beneficial in more than one population, even 748 when placed in the different genomic backgrounds of the two 749 subspecies. Our approach allowed us to distinguish between 750 multiple potential modes of convergence, including a neutral 751 model that models allele frequency covariance by drift alone 752 (Lee and Coop 2017). The distribution of most likely selection co- 753 efficients of the inferred beneficial alleles suggests the strength of 754 selection is moderate to strong, though this estimate is likely bi- 755 ased as strong positive selection will be easier to detect. Conver- 756 gence via independent mutations was by far the least frequent 757 mode. This is consistent with previous analyses of domestica- 758 tion (Hufford et al. 2012) and adaptation (Wang et al. 2020) in 759 maize, and unsurprising given evidence for ongoing gene flow 760 (Figure 1E), the relatively short evolutionary time scales, and the 761 low probability that even strongly selected new mutations can 762 overcome drift multiple times independently. When convergent 763 sweeps that occurred via standing variation within maize, or 764 for those shared between maize and teosinte, the distribution of 765 generation times that the selected variant was standing before 766 the onset of selection tended to be bimodal, with both long and 767 short standing times. Whereas sweeps exclusive to teosinte were 768 consistently inferred to be standing variation for more genera- 769 tions (Figure 5E). Sweeps that occurred via standing variation and shared between subspecies were often found in only a subset of maize populations. Many of these sweeps likely reflect the presence of structure in ancestral populations, suggesting different alleles beneficial to maize were likely derived from 771 more than one teosinte population.

The most common mode of convergent adaptation was via 773 migration, and frequently occurred between geographically des- 774 perate populations (Figures S4). This included a relatively large 775 number of shared sweeps via migration between maize and 776 teosinte (Figures 5 and S5). There is ample evidence that maize 777 and teosinte are capable of hybridizing (Wilkes et al. 1967; Ell- 778 strand et al. 2007; Ross-Ibarra et al. 2009). Gene flow between 779 geographically disparate populations has been inferred from 780 patterns of introgression between maize and Zea mays mexicana) 781 (Calfee et al. 2021). Further, convergence via migration between 782 geographically disparate maize populations has been inferred ${ }_{783}$ during adaptation to high elevations (Wang et al. 2020).

Our findings on convergence via migration point to an in- 785 triguing hypothesis, namely, that some non-trivial number of ${ }^{786}$ alleles that are beneficial to teosinte may have originally arisen ${ }^{787}$ in another teosinte population and moved between populations 788 via gene flow with maize, an idea suggested by Ross-lbarra et al. 789 (2009) based on allele sharing at a small set of loci. There are sev- 790 eral aspects of our results consistent with this hypothesis. Firstly, 791 we found relatively few shared sweeps exclusive to teosinte pop- 792 ulations (Figure 5C), which is what we would expect if maize 793 populations facilitate the movement of beneficial teosinte alle- 794 les. However, it is important to note that there are fewer sweeps 795 exclusive to teosinte for all modes of convergence, not just via mi- 796 gration, so this alone is not sufficient evidence. Secondly, sweeps 797 shared via migration that were exclusive to teosinte were always 798 inferred to be the lower of the two migration rates $\left(1 \times 10^{-3}\right){ }_{799}$ (Figure 5F), where those shared by both subspecies or exclusive 800 to maize were primarily inferred to be the higher migration 801 rate $\left(1 \times 10^{-1}\right)$. This indicates alleles that are only beneficial 802 in teosinte move between populations more slowly. Despite 803 the patterns of population structure in both subspecies, there is 804 evidence of gene flow based on $\mathrm{f} 4$ tests. In particular, f4 tests 805 that included maize from Crucero Lagunitas were consistently elevated across both subspecies (Figure 1E and S1). This is in accordance with our finding that Crucero Lagunitas maize was the second most commonly inferred source population of migration sweeps that were shared between maize and teosinte (Figure 5D). Together, these results suggests that geographically widespread varieties of maize such as Celaya (Crucero Lagunitas) (OrozcoRamírez et al. 2017) may have played a prominent role as a source of and/or transport for beneficial alleles among maize and teosinte populations. However, teosinte populations were more often the source of beneficial alleles for sweeps shared between both subspecies. In particular, the teosinte Palmar Chico was the most likely source population for the vast majority of sweeps shared between maize and teosinte as well as for sweeps exclusive to teosinte (Figure 5F). Palmar Chico is in the balsas region, near the putative site of maize domestication (Piperno et al. 2009), and our demographic inferences suggests Palmar Chico has maintained the largest effective population size of all the populations (Figure 2), meaning it is capable of generating the most mutations for selection to act on. As such, teosinte Palmar Chico and other populations in the Balsas region may have also been a particularly important source of adaptive variation for both teosinte and maize.

\section{Materials and Methods}

\section{Samples and whole genome re-sequencing}

We sampled seeds from five populations of Zea mays ssp. parviglumis, and four populations of Zea mays ssp. mays from plants growing across the species' native range. We additionally included populations of parviglumis and maize from Palmar Chico were previously analyzed and reported in (Chen et al. 2020) for a total of 6 and 5 populations of maize and teosinte (See Supplement I for accession IDs and further sample details). All maize and teosinte populations from each named location were less than $1 \mathrm{~km}$ from one another, with the exception of Crucero Lagunitas, which were separated by approximately $18 \mathrm{~km}$.

DNA extraction for teosinte followed (Chen et al. 2020). Genomic DNA for landraces was extracted from leaf tissue using the E.Z.N.A. ${ }^{\circledR}$ Plant DNA Kit (Omega Biotek), following manufacturer's instructions. DNA was quantified using Qubit (Life Technologies). 1ug of DNA per individual was fragmented using a bioruptor (Diagenode) with 30 seconds on/off cycles.

DNA fragments were then prepared for Illumina sequencing. First, DNA fragments were repaired with the End-Repair enzyme mix (New England Biolabs). A deoxyadenosine triphosphate was added at each $3^{\prime}$ end with the Klenow fragment (New England Biolabs). Illumina Truseq adapters (Affymetrix) were then added with the Quick ligase kit (New England Biolabs). Between each enzymatic step, DNA was washed with sera-mags speed beads (Fisher Scientific). The libraries were sequenced with an average coverage of 20 to $25 \mathrm{x}$ PE150 on the Xten at Novogene (Sacramento, USA).

We additionally grew one individual of Zea diploperennis from the UC Davis Botanical Conservatory as an outgroup. DNA for Zea diploperennis was extracted and libraries prepared as above, and then sequenced to 60X coverage using PE250 on 3 lanes of Illumina 2000 rapid run (UC Davis Genome Center, Davis, USA).

Sequencing reads have been deposited in the NCBI Sequence Read Archive under project number (to be submitted). 


\section{Sequencing and variant identification}

All paired-end reads were aligned to version 5 of the maize B73 reference genome (Hufford et al. 2021) using bwa-mem (v0.7.17) (Li 2013). Default 879 options were used for mapping except $\mathrm{M}$ to enable marking short hits 880 as secondary, $-\mathrm{R}$ for providing the read group, and $-\mathrm{K} 1000000$, for processing $10 \mathrm{Mb}$ input in each batch. Sentieon (v201808.01) (Freed et al. 881 2017) was used to process the alignments to remove duplicates (option 882 -algo Dedup) and to calculate various alignment metrics (GC bias, MQ value distribution, mean quality by cycle, and insert size metrics) to 883 ensure proper mapping of the reads.

All downstream analyses were based on genotype likelihoods estimated with ANGSD (v0.934) (Korneliussen et al. 2014) using the following command line flags and filters:

-GL 1

$-\mathrm{P} 5$

-uniqueOnly 1

-remove_bads 1

only_proper_pairs 1

-trim 0

-minMapQ 30

$-\min Q 30$

\section{Genetic Diversity}

We estimated per base nucleotide diversity $(\pi)$ and Tajima's $\mathrm{D}(D)$ in nonoverlapping $1 \mathrm{~kb}, 10 \mathrm{~kb}$ and $100 \mathrm{~kb}$ windows with thetaStat utility from $A N G S D$, though estimates did not substantively differ between window sizes. To estimate $\pi$ and the unfolded site frequency spectra for each 894 population, we polarized alleles as ancestral and derived using short- 895 read sequence data for Zea luxurians and Zea diploperennis as outgroups. 896 Zea luxurians sequence from Tenaillon et al. (2011) was downloaded 897 from The NCBI Sequence Read Archive (study SRR088692). We used 898 the alignments from the two species to make minor allele frequency 899 (MAF) files using ANGSD. We used the MAF files to construct a table 900 of genotypes found at each locus. Sites with minor allele frequency 901 estimates greater than 0.001 were treated as heterozygous. Sites that were 902 homozygous in both species were imputed onto the maize v5 reference 903 and assumed to be the ancestral allele. As there were substantially more 904 called bases in Zea luxurians than in Zea diploperennis, we also assumed 905 sites that were homozygous in luxurians and missing in diploperennis 906 were ancestral, but excluded sites that were missing from luxurians. 907 Sites that were classified as heterozygous were treated as missing and 908 imputed onto the maize reference as ' $\mathrm{N}$ '.

\section{Population Structure and Introgression}

We used ngsadmix (Skotte et al. 2013) to assess population structure within subspecies. To do so we used a SNP-calling procedure in ANGSD with the same filters as listed above, along with a SNP $p$ value cutoff of $1 \times 10^{-6}$. We looked for evidence of 911 gene flow between subspecies using $\mathrm{f} 4$ statistics and Z-scores calculated with blocked jackknifing, implemented using treemix (Pickrell and Pritchard 2012). Trees for f4 tests were always of the form (Maize_X, Maize_X; Teosinte_focal, Teosinte_X); each unique combination of populations was considered to be the "focal" and "_X" positions of the tree. We considered any tree with a Z-score greater than or equal to 3 significant, indicating a departure from the allele frequency differences expected if population history matched the hypothesized tree. We assessed Z-scores separately based on whether the focal population was maize or teosinte, and for trees that include the sympatric pair of the focal population.

\section{Demographic and Inbreeding History}

We inferred each population's demography using a single unfolded site frequency spectrum with mushi (v0.2.0) (DeWitt et al. 2021). In efforts to reduce overfitting given our modest samples sizes, we increased the regularization penalty parameters to alpha_tv $=1 \mathrm{e} 4$, alpha_spline $=1 \mathrm{e} 4$, and alpha_ridge $=1 \mathrm{e}-1$.

We assessed homozygosity by descent (HBD) in each population using IBDseq (v2.0) (Browning and Browning 2013). We compared empirical results to simulations in msprime (Kelleher et al. 2016) using each population's inferred demographic history. We performed ten 920 replicates of each of these simulations. Replicates were similar across all 921 populations; only one replicate was chosen at random for visual clarity. 922
We estimated recent inbreeding using ngsrelate (Hanghøj et al. 2019) with default parameters. Input files were generated using ANGSD with the same filters as listed above, along with a SNP $p$ value cutoff and maf filter of

$$
\begin{aligned}
& \text {-SNP_pval 1e-6 } \\
& \text {-minmaf } 0.05,
\end{aligned}
$$

respectively.

\section{Linked Selection}

Following an approach similar to Corbett-Detig et al. (2015), we modeled the effects of linked selection (background selection and hitchhiking) on observed values of $\pi$ across the genome in $1 \mathrm{~kb}$ windows.

The full model that jointly modeled both hitchhiking and background selections was

$$
\pi_{i}=\frac{\theta_{\text {neutral }}}{\left(1 / e^{-G_{i}}+\alpha * f d_{i} / R_{b p_{i}}\right)} .
$$

Here, $\alpha$ is the population-scaled rate of selective sweeps (Coop and Ralph 2012), $f d_{i}$ is the proportion of functional bases in window $i$, and $R_{b p_{i}}$ is the mean recombination rate per base pair in window $i$. Lastly, $G_{i}$ models the effects of background selection and is defined as,

$$
G_{i}=\Sigma_{k} \frac{U * f d_{k} * s h}{2 *\left(s h+P\left(\left|M_{i}-M_{k}\right|\right) *\left(s h+P\left(\left|M_{i}-M_{k}\right|\right)\right.\right.}
$$

where, $U$ is the deleterious mutation rate, $s$ and $h$ are the selection and dominance coefficients for deleterious mutations (respectively), $P$ is an index of panmixis, and $M_{i}$ and $M_{k}$ represent the Morgan position of the focal locus and all other loci along the chromosome, respectively (Hudson and Kaplan 1995). The proportion of functional bases $f d_{i}$, was estimated using the number of protein coding sequences in a window based on the most gene annotation of the maize v5 reference genome (Hufford et al. 2021). The position of loci in Morgans was approximated using the approx function in $\mathrm{R}$ based on a previously generated genetic map for maize that was lifted on to the v5 reference genome (Ogut et al 2015). We treated the parameters $s$ and $U$ as fixed, but refit the model over a grid of possible values for each. Specifically, we allowed $s$ to vary over 30 evenly spaced values between $1 \times 10^{-8}$ and $1 \times 10^{-1}, U$ took on values $1 \times 10^{-7}$ or $3 \times 10^{-8}$, while $P$ and $h$ were always fixed at 1 and 0.5 , respectively.

We compared the full linked selection model (equation 1 ) to three simpler models: one that does not account for hitchhiking

$$
\pi=\frac{\theta_{\text {neutral }}}{\left(1 / e^{-G_{i}}\right)},
$$

one that does not include background selection,

$$
\pi=\frac{\theta_{\text {neutral }}}{\left.1+\alpha * f d_{i} / R_{b p_{i}}\right)}
$$

and finally a simple intercept model that estimates neutral $\theta$

$$
\pi=\theta_{\text {neutral }} .
$$

The same refitting procedure was used for all models that included $U$, $s$, and $h$. We fit all four equations separately for each population using a Maximum Likelihood framework. Since all values of $\theta$ are bounded between $(0,1)$, we assumed observations were generated from a beta distribution following

$$
\mathbf{L}=\prod_{i} \operatorname{bet} a\left(\pi_{i} * \sigma,\left(1-\pi_{i}\right) * \sigma\right) .
$$

We calculated the standard errors for parameters from the Hessian matrix (H) produced by the optim function as

$$
C I_{\text {parameter }}=\text { parameter } \pm 1.96 * \sqrt{\operatorname{diag}\left(\mathbf{H}^{-1}\right)} .
$$

We calculated AIC, $\triangle A I C$ and AIC weights (Wagenmakers and Farrell 2004) and used them to compare among the alternative models for each population. 


\section{Estimating the Rate of Positive Selection, $\alpha$}

We modeled the rate of positive selection, $\alpha$ (note this is different from 983 the $\alpha$ estimated in our linked selection models above), of 0 -fold non- ${ }^{984}$ synonymous mutations using the Asymptotic extension of the McDon- 985 ald-Kreitman (MK) test (Messer and Petrov 2013), where $\alpha$ is calculated 9 at each allele frequency bin of the uSFS (from $1 / n$ to $(n-1) / n$ ). At each allele frequency bin, each $\alpha$ was calculated as

$$
\alpha=1-\frac{d_{0}}{d} \frac{p}{p_{0}}
$$

where $d_{0}$ and $d$ are the number of derived fixed differences for selected and putatively neutral sites, respsectively, and $p_{0}$ and $p$ are the number of selected and putatively neutral polymorphic sites. We identified 0fold and 4-fold sites using Python (https://github.com/silastittes/cds_fold). We fit the Asymptotic MK extension as a nonlinear Bayesian mixedmodel using the R package brms (Bürkner 2017, 2018).

$$
\alpha_{i j} \sim a_{j}+b_{j} e^{-c_{j} x_{i j}}
$$

where $\alpha_{i j}$ is the value of $\alpha$ calculated at the $i^{t h}$ allele frequency bin of the $j^{\text {th }}$ population and $x_{i j}$ is the corresponding allele frequency bin. The nonlinear brms model was coded as

$$
\begin{aligned}
\text { alpha } & \sim \\
a & +b * \exp \left(-c * a l l e l e_{\text {ffrequency }}\right)
\end{aligned}
$$

where all three free parameters of the asymptotic function $(a, b$, and $c)$ were treated as random effects of population and nucleotide type (see Supplement IV), and the subspecies was treated as a fixed effect. These effects were coded in brms as

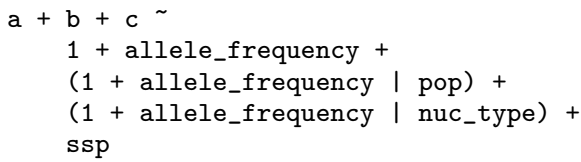

\section{Identifying Selective Sweeps}

We used RAiSD (Alachiotis and Pavlidis 2018) to infer regions that ${ }^{1015}$ have experienced a selective sweep in each population, including the ${ }_{1017}$ rangewide samples. Across all populations, we used a minor allele frequency threshold of 0.05 and a window size of 100 SNPs. We called ${ }_{1019}$ SNPs and generated VCF files for each population using the dovcf utility from ANGSD, using a SNP calling $\mathrm{p}$ value cut off of $1 \times 10^{-6}$. We used the Python package mop (https://pypi.org/project/mop-bam/) to exclude SNPs that fell in regions with low and excessive coverage and/or poor 1022 quality. Here we required each locus to have at least $70 \%$ of individuals ${ }^{1023}$ with a depth between 5 and 100, and to have a phred scaled quality scores ${ }^{1024}$ above 30 for base and mapping quality. We additionally used mop to 1025 rescale the $\mu_{\text {var }}$ sub-statistic in each population based on the proportion ${ }^{1026}$ of high quality bases available in each of the RAiSD SNP windows, ${ }^{1027}$ after which we recalculated the overall $\mu$ statistic as the product of ${ }^{1028}$ the three sub-statistics (Alachiotis and Pavlidis 2018). After correcting ${ }_{102}$ the $\mu$ statistic, we defined outliers using simulations of demography ${ }_{1030}$ using msprime (Kelleher et al. 2016). We considered estimates of $\mu_{1031}$ that were greater than the $99.99 \%$ quantile of the neutral simulations ${ }_{1032}$ as evidence for being within a sweep region. RAiSD outliers within $100 \mathrm{~kb}$ were merged using Bedtools merge (Quinlan and Hall 2010) and ${ }_{1034}$ treated as a single sweep region in downstream analyses. We then used Bedtools intersect to identify overlapping sweep regions among ${ }_{1036}$ populations which we treated as evidence for being a shared sweep.

Assessing the number of false positive sweeps and the number of shared sweeps expected by chance

To assess false positives, we used a second random sample non- 104 overlapping individuals from the two Palmar Chico populations. False positives were assessed based on the number of sweep regions that did not overlap between the 2 replicate Palmar Chico samples from each subspecies. To account for differences in the total number of sweep 1043 regions for each replicate, we averaged the two proportions

$$
P=\left(\frac{n_{S}}{n_{P 1}}+\frac{n_{S}}{n_{P 2}}\right) / 2
$$

and

$$
F P=1-P
$$

where $n_{S}$ is the number of sweeps shared between the replicates, and $n_{P 1}$ and $n_{P 2}$ were the number of sweeps in the first and second replicates, respectively. In downstream analyses, we used the average value of $F P$ for the two subspecies, although it was higher for maize than for teosinte ( 0.60 and 0.49 for maize and teosinte, respectively).

We evaluated the number of sweeps that we would expect populations to share by chance using a simple statistical test based on the hypergeometric distribution,

$$
\operatorname{Pr}(x \geq X)=\sum_{x \geq X}^{n_{1}} \frac{\left(\begin{array}{c}
n_{2} \\
x
\end{array}\right)\left(\begin{array}{c}
N \\
n_{1}-x
\end{array}\right)}{\left(\begin{array}{c}
N \\
n_{1}
\end{array}\right)}
$$

where $N$ is the total number of loci tested; $n_{1}$ and $n_{2}$ are the number of outlier loci in the first and second populations, respectively; and $x$ is the number of shared outliers between the two populations. The population with the larger number of outliers was always designated at the first population. We accounted for false positive by multiplying the raw values of $N, n_{1}, n_{2}$, and $x$ by the $F P$ value described above.

We corrected p-values for multiple tests using the Benjamini and Yekutieli method, implemented with the R function, p.adjust (Benjamini and Yekutieli 2001).

\section{Inferring modes of convergent adaptation}

For sweep regions that were overlapping in 2 to 9 of the 11 populations, we used $r d m c$ to infer the most likely mode of convergent adaptation (Lee and Coop 2017; Tittes 2020). To ensure a sufficient number loci were included to estimate decay in covariance across the sweep regions, we added $10 \%$ of each sweep region's total length on each of its ends prior to fitting the models. To reduce the computation time, we exclude sites that had an allele frequency less than $1 / 20$ across all populations. As sweep regions differed in size, we subset from their total number of sites to maintain approximately 250K SNPs per centiMorgan. All sweeps regions were required to have fewer than 100K SNPs. Sweep regions near the ends of chromosomes for which we could not estimate the number of centiMorgans for were subset to $10 \mathrm{~K} \mathrm{SNPs.} \mathrm{To} \mathrm{contrast}$ allele frequency covariance in sweep regions, we used allele frequencies at $100 \mathrm{~K}$ random loci to estimate the neutral covariance among all populations. To reduce computation time when fitting the models, we estimated a covariance matrix using a random subset of $50 \mathrm{~K}$ of these loci. When fitting $r d m c$, we assumed the effective population size was $50 \mathrm{~K}$. The recombination rate was approximated for each sweep region as the median interpolated value based on a previously generated genetic map for maize that was lifted on to the $\mathrm{v} 5$ reference genome (Ogut et al. 2015). The $r d m c$ function arguments that control the grid of parameter values over which composite likelihood were computed were:

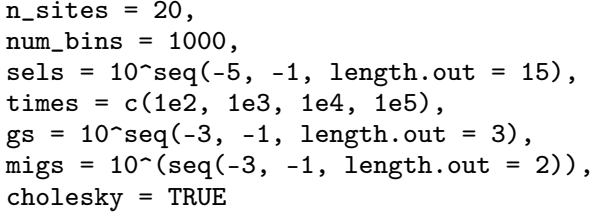

We compared composite likelihoods over four convergent adaptation modes, "neutral", "independent", "standing", and "migration". We assigned each sweep to the mode with the highest log composite likelihood. To assess the overall performance of the method to distinguish between the four modes, we computed differences between the highest composite likelihood and the next highest for each sweep.

All wrangling to prepare input data for statistical analyses was done using $R$ (R Core Team 2020) with appreciable reliance on functions from the tidyverse package suite (Wickham et al. 2019). Figures were made using ggplot2 (Wickham 2016), patchwork (Pedersen 2019), and cowplot (Wilke 2019). Code and summarised data for the entirety of the analyses, including Jupyter notebooks for reproducing figures, is available from https://github.com/silastittes/parv_local permanent repository to follow.

\section{Acknowledgments}

We would like to thank Andi Kur for providing the corn art, along with Matthew Gibson, Tom Booker, Cathy Rushworth, and other members of the Ross-Ibarra lab for feedback and suggestions on early drafts of the manuscript. This work was funded in part by grants from the National Science Foundation (1822330 and 1238014). We would also like to acknowledge Felix Andrews for statistical advice, although we did not follow it. 


\section{References}

Aguirre-Liguori, J. A. B. S. Gaut J. P. Jaramillo-Correa, M. I. Tenaillor S. Montes-Hernández, et al., 2019 Divergence with gene flow is driven ${ }^{1128}$ by local adaptation to temperature and soil phosphorus concentration ${ }^{1129}$ in teosinte subspecies (zea mays parviglumis and zea mays mexicana). ${ }^{1130}$ Molecular ecology 28: 2814-2830.

Alachiotis, N. and P. Pavlidis, 2018 Raisd detects positive selection based ${ }^{1132}$ on multiple signatures of a selective sweep and snp vectors. Commu- ${ }^{1133}$ nications biology 1: 1-11.

Beissinger, T. M., L. Wang, K. Crosby, A. Durvasula, M. B. Hufford, et al, 1135 2016 Recent demography drives changes in linked selection across ${ }^{1136}$ the maize genome. Nature plants 2: 1-7.

Bellon, M. R., A. Mastretta-Yanes, A. Ponce-Mendoza, D. Ortiz- ${ }^{1138}$ Santamaría, O. Oliveros-Galindo, et al., 2018 Evolutionary and food ${ }^{1139}$ supply implications of ongoing maize domestication by mexican ${ }^{1140}$ campesinos. Proceedings of the Royal Society B 285: 20181049.

Benjamini, Y. and D. Yekutieli, 2001 The control of the false discovery ${ }^{1142}$ rate in multiple testing under dependency. Annals of statistics pp. ${ }^{1143}$ $1165-1188$

Bourne, E. C., G. Bocedi, J. M. Travis, R. J. Pakeman, R. W. Brooker, ${ }^{1145}$ et al., 2014 Between migration load and evolutionary rescue: dispersal, ${ }^{1146}$ adaptation and the response of spatially structured populations to ${ }^{1147}$ environmental change. Proceedings of the Royal Society B: Biological ${ }^{1148}$ Sciences 281: 20132795.

Bragg, J. G., M. A. Supple, R. L. Andrew, and J. O. Borevitz, 2015 Ge- 1150 nomic variation across landscapes: insights and applications. New ${ }^{1151}$ Phytologist 207: 953-967.

Browning, B. L. and S. R. Browning, 2013 Detecting identity by descent ${ }^{1153}$ and estimating genotype error rates in sequence data. The American ${ }^{1154}$ Journal of Human Genetics 93: 840-851.

Buckler, E. and T. P. Holtsford, 1996 Zea systematics: ribosomal its ${ }^{115}$ evidence. Molecular Biology and Evolution 13: 612-622.

Buffalo, V., 2021 Why do species get a thin slice of $\pi$ ? revisiting lewon- ${ }^{115}$ tin's paradox of variation. bioRxiv .

Butler, E. E. and P. Huybers, 2013 Adaptation of us maize to temperature 1160 variations. Nature Climate Change 3: 68-72.

Bürkner, P.-C., 2017 brms: An R package for Bayesian multilevel models ${ }^{116}$ using Stan. Journal of Statistical Software 80: 1-28.

Bürkner, P.-C., 2018 Advanced Bayesian multilevel modeling with the R ${ }^{116}$ package brms. The R Journal 10: 395-411.

Calfee, E., D. Gates, A. Lorant, M. T. Perkins, G. Coop, et al., 2021 Selec- ${ }^{1166}$ tive sorting of ancestral introgression in maize and teosinte along an ${ }^{1167}$ elevational cline. bioRxiv .

Charlesworth, B., 2020 How long does it take to fix a favorable mutation, 1169 and why should we care? The American Naturalist 195: 753-771. ${ }_{1170}$

Chen, Q., L. F. Samayoa, C. J. Yang, P. J. Bradbury, B. A. Olukolu, et al., 1171 2020 The genetic architecture of the maize progenitor, teosinte, and 1172 how it was altered during maize domestication. PLoS genetics 16: ${ }^{1173}$ e1008791.

Chevin, L.-M., G. Martin, and T. Lenormand, 2010 Fisher's model and the ${ }^{1175}$ genomics of adaptation: restricted pleiotropy, heterogenous mutation, ${ }^{1176}$ and parallel evolution. Evolution: International Journal of Organic ${ }^{1177}$ Evolution 64: 3213-3231.

Clark, R. M., S. Tavaré, and J. Doebley, 2005 Estimating a nucleotide sub- 1179 stitution rate for maize from polymorphism at a major domestication ${ }^{1180}$ locus. Molecular biology and evolution 22: 2304-2312.

Clausen, J., D. D. Keck, W. M. Hiesey, et al., 1948 Experimental studies on ${ }^{1182}$ the nature of species. iii. environresponses of climatic races of achillea. ${ }^{1183}$ Experimental studies on the nature of species. III. Environresponses ${ }^{1184}$ of climatic races of Achillea.

Colosimo, P. F., K. E. Hosemann, S. Balabhadra, G. Villarreal, M. Dickson, ${ }^{1186}$ et al., 2005 Widespread parallel evolution in sticklebacks by repeated ${ }^{1187}$ fixation of ectodysplasin alleles. science 307: 1928-1933.

Coop, G. and P. Ralph, 2012 Patterns of neutral diversity under general 1189 models of selective sweeps. Genetics 192: 205-224.

Corbett-Detig, R. B., D. L. Hartl, and T. B. Sackton, 2015 Natural selection ${ }^{1191}$ constrains neutral diversity across a wide range of species. PLoS Biol ${ }^{1192}$ 13: e1002112.

Coventry, A., L. M. Bull-Otterson, X. Liu, A. G. Clark, T. J. Maxwell, et al., 1194 2010 Deep resequencing reveals excess rare recent variants consistent ${ }^{1195}$ with explosive population growth. Nature communications 1: 1-6. ${ }_{1196}$

DeWitt, W. S., K. D. Harris, A. P. Ragsdale, and K. Harris, 2021 Non- ${ }^{1197}$ parametric coalescent inference of mutation spectrum history and ${ }^{1198}$ demography. Proceedings of the National Academy of Sciences 118. ${ }^{1199}$ Doebley, J., 1989 Isozymic evidence and the evolution of crop plants. In ${ }^{1200}$
Isozymes in plant biology, pp. 165-191, Springer.

Ellstrand, N. C., L. C. Garner, S. Hegde, R. Guadagnuolo, and L. Blancas, 2007 Spontaneous hybridization between maize and teosinte. Journal of Heredity 98: 183-187.

Eyre-Walker, A., R. L. Gaut, H. Hilton, D. L. Feldman, and B. S. Gaut, 1998 Investigation of the bottleneck leading to the domestication of maize. Proceedings of the National Academy of Sciences 95: 44414446.

Fournier-Level, A., A. Korte, M. D. Cooper, M. Nordborg, J. Schmitt, et al., 2011 A map of local adaptation in arabidopsis thaliana. Science 334: 86-89.

Freed, D., R. Aldana, J. A. Weber, and J. S. Edwards, 2017 The sentieon genomics tools-a fast and accurate solution to variant calling from next-generation sequence data. BioRxiv p. 115717.

Fustier, M.-A., J.-T. Brandenburg, S. Boitard, J. Lapeyronnie, L. Eguiarte, et al., 2017 Signatures of local adaptation in lowland and highland teosintes from whole-genome sequencing of pooled samples. Molecular Ecology 26: 2738-2756.

Fustier, M.-A., N. E. Martínez-Ainsworth, J. A. Aguirre-Liguori, A. Venon, H. Corti, et al., 2019 Common gardens in teosintes reveal the establishment of a syndrome of adaptation to altitude. PLoS genetics 15: e1008512.

Galtier, N., 2016 Adaptive protein evolution in animals and the effective population size hypothesis. PLoS genetics 12: e1005774.

Gates, D. J., D. Runcie, G. M. Janzen, A. R. Navarro, M. Willcox, et al., 2019 Single-gene resolution of locally adaptive genetic variation in mexican maize. BioRxiv p. 706739.

Gillespie, J. H., 2004 Population genetics: a concise guide. JHU Press.

Gossmann, T. I., B.-H. Song, A. J. Windsor, T. Mitchell-Olds, C. J. Dixon, et al., 2010 Genome wide analyses reveal little evidence for adaptive evolution in many plant species. Molecular biology and evolution 27: 1822-1832.

Haines, M. R., M. R. Haines, and R. H. Steckel, 2000 A population history of North America. Cambridge University Press.

Haller, B. C. and P. W. Messer, 2019 Slim 3: forward genetic simulations beyond the wright-fisher model. Molecular biology and evolution 36: 632-637.

Hämälä, T. and P. Tiffin, 2020 Biased gene conversion constrains adaptation in arabidopsis thaliana. Genetics 215: 831-846.

Hamrick, J. and R. W. Allard, 1972 Microgeographical variation in allozyme frequencies in avena barbata. Proceedings of the National Academy of Sciences 69: 2100-2104.

Hanghøj, K., I. Moltke, P. A. Andersen, A. Manica, and T. S. Korneliussen, 2019 Fast and accurate relatedness estimation from high-throughput sequencing data in the presence of inbreeding. Gigascience 8: giz034. Hoban, S., J. L. Kelley, K. E. Lotterhos, M. F. Antolin, G. Bradburd, et al., 2016 Finding the genomic basis of local adaptation: pitfalls, practical solutions, and future directions. The American Naturalist 188: 379397.

Hudson, R. R. and N. L. Kaplan, 1995 Deleterious background selection with recombination. Genetics 141: 1605-1617.

Hufford, M. B., 2010 Genetic and ecological approaches to guide conservation of teosinte (Zea mays ssp. parviglumis), the wild progenitor of maize. University of California, Davis.

Hufford, M. B., A. S. Seetharam, M. R. Woodhouse, K. M. Chougule, S. Ou, et al., 2021 De novo assembly, annotation, and comparative analysis of 26 diverse maize genomes. Science .

Hufford, M. B., X. Xu, J. Van Heerwaarden, T. Pyhäjärvi, J.-M. Chia, et al., 2012 Comparative population genomics of maize domestication and improvement. Nature genetics 44: 808.

Keinan, A. and A. G. Clark, 2012 Recent explosive human population growth has resulted in an excess of rare genetic variants. science 336 : 740-743.

Kelleher, J., A. M. Etheridge, and G. McVean, 2016 Efficient coalescent simulation and genealogical analysis for large sample sizes. PLoS computational biology 12: e1004842.

Korneliussen, T. S., A. Albrechtsen, and R. Nielsen, 2014 Angsd: analysis of next generation sequencing data. BMC bioinformatics 15: 356 .

Lee, K. M. and G. Coop, 2017 Distinguishing among modes of convergent adaptation using population genomic data. Genetics 207: 1591-1619.

Levy, R. and E. Borenstein, 2012 Reverse ecology: from systems to environments and back. In Evolutionary systems biology, pp. 329-345, Springer.

Lewontin, R. C. et al., 1974 The genetic basis of evolutionary change, volume 560. Columbia University Press New York.

Li, H., 2013 Aligning sequence reads, clone sequences and assembly 
bioRxiv preprint doi: https://doi.org/10.1101/2021.09.09.459637; this version posted September 10, 2021. The copyright holder for this preprint (which was not certified by peer review) is the author/funder, who has granted bioRxiv a license to display the preprint in perpetuity. It is made available under aCC-BY 4.0 International license.

contigs with bwa-mem. arXiv preprint arXiv:1303.3997 .

Li, Y. F., J. C. Costello, A. K. Holloway, and M. W. Hahn, 2008 "reverse 1278 ecology" and the power of population genomics. Evolution: Interna- 1279 tional Journal of Organic Evolution 62: 2984-2994.

Lowry, D. B., R. C. Rockwood, and J. H. Willis, 2008 Ecological reproduc- 128 tive isolation of coast and inland races of mimulus guttatus. Evolution: 1282 International Journal of Organic Evolution 62: 2196-2214.

Mei, W., M. G. Stetter, D. J. Gates, M. C. Stitzer, and J. Ross-Ibarra, 20181284 Adaptation in plant genomes: Bigger is different.

Messer, P. W. and D. A. Petrov, 2013 Frequent adaptation and the 1286 mcdonald-kreitman test. Proceedings of the National Academy of ${ }_{1287}$ Sciences 110: 8615-8620.

Nannas, N. J. and R. K. Dawe, 2015 Genetic and genomic toolbox of zea 1289 mays. Genetics 199: 655-669.

O’Brien, A. M., R. J. Sawers, S. Y. Strauss, and J. Ross-Ibarra, 2019 Adap- 1291 tive phenotypic divergence in an annual grass differs across biotic ${ }_{1292}$ contexts. Evolution 73: 2230-2246.

Ogut, F., Y. Bian, P. J. Bradbury, and J. B. Holland, 2015 Joint-multiple ${ }_{1294}^{1293}$ family linkage analysis predicts within-family variation better than ${ }_{1295}$ single-family analysis of the maize nested association mapping popu- ${ }_{1296}$ lation. Heredity 114: 552-563.

Orozco-Ramírez, Q., H. Perales, and R. J. Hijmans, 2017 Geographical ${ }_{1298}$ distribution and diversity of maize (zea mays l. subsp. mays) races in ${ }_{1299}$ mexico. Genetic resources and crop evolution 64: 855-865.

Ossowski, S., K. Schneeberger, J. I. Lucas-Lledó, N. Warthmann, R. M. ${ }^{1300}$ Clark, et al., 2010 The rate and molecular spectrum of spontaneous mutations in arabidopsis thaliana. science 327: 92-94.

Pedersen, T. L., 2019 patchwork: The Composer of Plots. R package version ${ }_{1304}^{130}$ 1.0.0.

Pickrell, J. and J. Pritchard, 2012 Inference of population splits and ${ }^{1305}$ mixtures from genome-wide allele frequency data. Nature Precedings ${ }_{1307}$ pp. 1-1.

Piperno, D. R., 1991 The status of phytolith analysis in the american ${ }_{1309}^{1308}$ tropics. Journal of World Prehistory 5: 155-191.

Piperno, D. R., A. J. Ranere, I. Holst, J. Iriarte, and R. Dickau, $2009{ }_{13}^{13}$ Starch grain and phytolith evidence for early ninth millennium bp ${ }_{1312}^{1311}$ maize from the central balsas river valley, mexico. Proceedings of the National Academy of Sciences 106: 5019-5024.

Portwood, J. L., M. R. Woodhouse, E. K. Cannon, J. M. Gardiner, L. C. ${ }^{1314}$ Harper, et al., 2019 Maizegdb 2018: the maize multi-genome genetics ${ }_{1316}$ and genomics database. Nucleic acids research 47: D1146-D1154.

Pritchard, J. K., J. K. Pickrell, and G. Coop, 2010 The genetics of human ${ }^{1317}$ adaptation: hard sweeps, soft sweeps, and polygenic adaptation. ${ }_{1319}{ }_{1318}$ Current biology 20: R208-R215.

Pyhäjärvi, T., M. B. Hufford, S. Mezmouk, and J. Ross-Ibarra, 2013 Complex patterns of local adaptation in teosinte. Genome biology and evolution 5: 1594-1609.

Quinlan, A. R. and I. M. Hall, 2010 Bedtools: a flexible suite of utilities for comparing genomic features. Bioinformatics 26: 841-842.

R Core Team, 2020 R: A Language and Environment for Statistical Computing. $\mathrm{R}$ Foundation for Statistical Computing, Vienna, Austria.

Ranum, P., J. P. Peña-Rosas, and M. N. Garcia-Casal, 2014 Global maize production, utilization, and consumption. Annals of the new York academy of sciences 1312: 105-112.

Rodriguez-Zapata, F., A. C. Barnes, K. A. Blocher-Juarez, D. J. Gates, A. Kur, et al., 2021 Teosinte introgression modulates phosphatidylcholine levels and induces early maize flowering time. bioRxiv .

Ross-Ibarra, J., M. Tenaillon, and B. S. Gaut, 2009 Historical divergence and gene flow in the genus zea. Genetics 181: 1399-1413.

Rudman, S. M., S. I. Greenblum, S. Rajpurohit, N. J. Betancourt, J. Hanna, et al., 2021 Direct observation of adaptive tracking on ecological timescales in drosophila. bioRxiv

Savolainen, O., M. Lascoux, and J. Merilä, 2013 Ecological genomics of local adaptation. Nature Reviews Genetics 14: 807-820.

Schrider, D. R. and A. D. Kern, 2016 S/hic: robust identification of soft and hard sweeps using machine learning. PLoS genetics 12: e1005928.

Skotte, L., T. S. Korneliussen, and A. Albrechtsen, 2013 Estimating individual admixture proportions from next generation sequencing data. Genetics 195: 693-702.

Smith, N. G. and A. Eyre-Walker, 2002 Adaptive protein evolution in drosophila. Nature 415: 1022-1024.

Stitzer, M. C. and J. Ross-Ibarra, 2018 Maize domestication and gene interaction. New phytologist 220: 395-408.

Swarts, K., R. M. Gutaker, B. Benz, M. Blake, R. Bukowski, et al., 2017 Genomic estimation of complex traits reveals ancient maize adaptation to temperate north america. Science 357: 512-515.
Tenaillon, M. I., M. B. Hufford, B. S. Gaut, and J. Ross-Ibarra, 2011 Genome size and transposable element content as determined by high-throughput sequencing in maize and zea luxurians. Genome biology and evolution 3: 219-229.

Tittes, S., 2020 rdmc: an open source $r$ package implementing convergent adaptation models of lee and coop (2017). G3: Genes, Genomes, Genetics 10: 3041-3046.

Tuanmu, M.-N. and W. Jetz, 2015 A global, remote sensing-based characterization of terrestrial habitat heterogeneity for biodiversity and ecosystem modelling. Global Ecology and Biogeography 24: 1329_ 1339.

Uricchio, L. H., D. A. Petrov, and D. Enard, 2019 Exploiting selection at linked sites to infer the rate and strength of adaptation. Nature ecology \& evolution 3: 977-984.

Van Heerwaarden, J., J. ROSS-IBARRA, J. Doebley, J. C. Glaubitz, J. DE JESÚS SÁNCHEZ GONZÁLEZ, et al., 2010 Fine scale genetic structure in the wild ancestor of maize (zea mays ssp. parviglumis). Molecular Ecology 19: 1162-1173.

Wagenmakers, E.-J. and S. Farrell, 2004 Aic model selection using akaike weights. Psychonomic bulletin \& review 11: 192-196.

Wang, L., T. M. Beissinger, A. Lorant, C. Ross-Ibarra, J. Ross-Ibarra, et al., 2017 The interplay of demography and selection during maize domestication and expansion. Genome biology 18: 1-13.

Wang, L., E. B. Josephs, K. M. Lee, L. M. Roberts, R. Rellán-Álvarez, et al., 2020 Molecular parallelism underlies convergent highland adaptation of maize landraces. bioRxiv

Whitehead, A., F. Galvez, S. Zhang, L. M. Williams, and M. F. Oleksiak, 2011 Functional genomics of physiological plasticity and local adaptation in killifish. Journal of Heredity 102: 499-511.

Wickham, H., 2016 ggplot2: Elegant Graphics for Data Analysis. SpringerVerlag New York.

Wickham, H., M. Averick, J. Bryan, W. Chang, L. D. McGowan, et al., 2019 Welcome to the tidyverse. Journal of Open Source Software 4: 1686.

Wilke, C. O., 2019 cowplot: Streamlined Plot Theme and Plot Annotations for 'ggplot2'. R package version 1.0.0.

Wilkes, H. G. et al., 1967 Teosinte: The closest relative of maize. Teosinte: the closest relative of maize.

Wright, S. I., I. V. Bi, S. G. Schroeder, M. Yamasaki, J. F. Doebley, et al., 2005 The effects of artificial selection on the maize genome. Science 308: 1310-1314.

Xue, A. T., D. R. Schrider, and A. D. Kern, 2021 Discovery of ongoing selective sweeps within anopheles mosquito populations using deep learning. Molecular biology and evolution 38: 1168-1183. 
bioRxiv preprint doi: https://doi.org/10.1101/2021.09.09.459637; this version posted September 10, 2021. The copyright holder for this preprint (which was not certified by peer review) is the author/funder, who has granted bioRxiv a license to display the preprint in perpetuity. It is made available under aCC-BY 4.0 International license.

1321 Supplement I

${ }_{1322}$

Population sampling locations

Table S1 Population sampling location information.

\begin{tabular}{lcccccc}
\hline Population & Subspecies & Sample size & Latitude & Longitude & Elevation (meters) & Accession ID \\
\hline \hline Crucero Lagunitas & Maize & 10 & 16.98 & -99.28 & 201 & $2373-G R O-294$ \\
Amatlán de Cañas & Maize & 10 & 20.82 & -104.41 & 760 & $5054-N A Y-310$ \\
Los Guajes & Maize & 10 & 19.23 & -100.49 & 985 & TC-300 \\
San Lorenzo & Maize & 10 & 19.94 & -103.99 & 982 & RMM-15 \\
Palmar Chico & Maize & 55 & 18.64 & -100.35 & 1008 & JSG-RMM-LCL-529 \\
Crucero Lagunitas & Teosinte & 10 & 16.85 & -99.06 & 590 & JSG-RMM-LCL-487 \\
Amatlán de Cañas & Teosinte & 10 & 20.82 & -104.41 & 880 & JSG-JRP-ERG-543 \\
El Rodeo & Teosinte & 10 & 16.35 & -97.02 & 982 & JSG-RMM-LCL-486 \\
Los Guajes & Teosinte & 10 & 19.23 & -100.49 & 851 & JSG Y RMM-454 \\
San Lorenzo & Teosinte & 10 & 19.94 & -103.99 & 982 & RMM-13 \\
Palmar Chico & Teosinte & 50 & 18.64 & -100.35 & 983 & JSG-RMM-LCL-528 \\
\hline
\end{tabular}




\section{Supplement II}

\section{Further assessment of $\mathbf{f} 4$ statistic inferences}

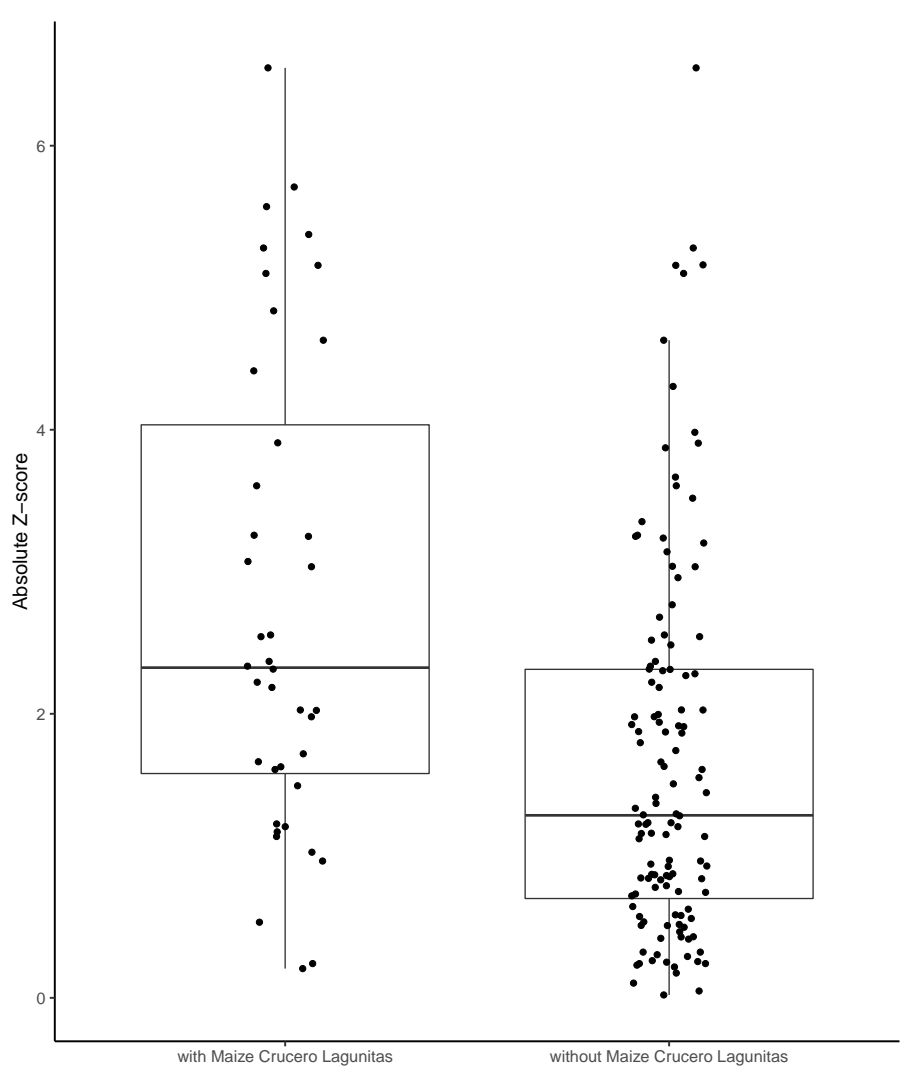

Figure S1 $\mathrm{f} 4$ tests including the maize Crucero Lagunitas population are significantly elevated compared to those without.

Table S2 Significant F4 tests. Each row of the table reports the number of significant $f 4$ tests that occurred with a given focal and secondary population, where the two other tip positions were filled with each of the remaining populations for each subspecies. Rows that are left blank in the secondary column are used to report the total number of significant trees for a given focal population.

\begin{tabular}{lll} 
Focal population & Secondary population & Count \\
\hline Maize Amatlan de Canas & & 5 \\
Maize Amatlan de Canas & Maize Crucero Lagunitas & 5 \\
Maize Amatlan de Canas & Teosinte Amatlan de Canas & 3 \\
Maize Amatlan de Canas & Teosinte El Rodeo & 2 \\
Maize Amatlan de Canas & Teosinte Palmar Chico & 2 \\
Maize Amatlan de Canas & Teosinte San Lorenzo & 2 \\
Maize Amatlan de Canas & Teosinte Los Guajes & 1 \\
\hline Maize Crucero Lagunitas & & 15 \\
Maize Crucero Lagunitas & Teosinte Amatlan de Canas & 9 \\
Maize Crucero Lagunitas & Teosinte Crucero Lagunitas & 6 \\
Maize Crucero Lagunitas & Teosinte El Rodeo & 6 \\
Maize Crucero Lagunitas & Maize Palmar Chico & 5 \\
Maize Crucero Lagunitas & Maize Los Guajes & 4 \\
Maize Crucero Lagunitas & Teosinte San Lorenzo & 4
\end{tabular}


bioRxiv preprint doi: https://doi.org/10.1101/2021.09.09.459637; this version posted September 10, 2021. The copyright holder for this preprint (which was not certified by peer review) is the author/funder, who has granted bioRxiv a license to display the preprint in perpetuity. It is made available under aCC-BY 4.0 International license.

\begin{tabular}{|c|c|c|}
\hline Maize Crucero Lagunitas & Maize Amatlan de Canas & 3 \\
\hline Maize Crucero Lagunitas & Maize San Lorenzo & 3 \\
\hline Maize Crucero Lagunitas & Teosinte Palmar Chico & 3 \\
\hline Maize Crucero Lagunitas & Teosinte Los Guajes & 2 \\
\hline Maize Los Guajes & & 6 \\
\hline Maize Los Guajes & Teosinte Amatlan de Canas & 4 \\
\hline Maize Los Guajes & Maize Crucero Lagunitas & 3 \\
\hline Maize Los Guajes & Teosinte Crucero Lagunitas & 3 \\
\hline Maize Los Guajes & Maize San Lorenzo & 2 \\
\hline Maize Los Guajes & Teosinte Palmar Chico & 2 \\
\hline Maize Los Guajes & Maize Palmar Chico & 1 \\
\hline Maize Los Guajes & Teosinte El Rodeo & 1 \\
\hline Maize Los Guajes & Teosinte Los Guajes & 1 \\
\hline Maize Los Guajes & Teosinte San Lorenzo & 1 \\
\hline Maize Palmar Chico & & 9 \\
\hline Maize Palmar Chico & Teosinte Amatlan de Canas & 7 \\
\hline Maize Palmar Chico & Maize Crucero Lagunitas & 5 \\
\hline Maize Palmar Chico & Teosinte Palmar Chico & 4 \\
\hline Maize Palmar Chico & Teosinte El Rodeo & 3 \\
\hline Maize Palmar Chico & Maize Los Guajes & 2 \\
\hline Maize Palmar Chico & Maize San Lorenzo & 2 \\
\hline Maize Palmar Chico & Teosinte San Lorenzo & 2 \\
\hline Maize Palmar Chico & Teosinte Crucero Lagunitas & 1 \\
\hline Maize Palmar Chico & Teosinte Los Guajes & 1 \\
\hline Maize San Lorenzo & & 6 \\
\hline Maize San Lorenzo & Teosinte Amatlan de Canas & 4 \\
\hline Maize San Lorenzo & Maize Crucero Lagunitas & 3 \\
\hline Maize San Lorenzo & Maize Los Guajes & 2 \\
\hline Maize San Lorenzo & Teosinte Crucero Lagunitas & 2 \\
\hline Maize San Lorenzo & Teosinte El Rodeo & 2 \\
\hline Maize San Lorenzo & Teosinte Palmar Chico & 2 \\
\hline Maize San Lorenzo & Maize Palmar Chico & 1 \\
\hline Maize San Lorenzo & Teosinte Los Guajes & 1 \\
\hline Maize San Lorenzo & Teosinte San Lorenzo & 1 \\
\hline Teosinte Amatlan de Canas & & 11 \\
\hline Teosinte Amatlan de Canas & Maize Crucero Lagunitas & 9 \\
\hline Teosinte Amatlan de Canas & Maize Palmar Chico & 4 \\
\hline Teosinte Amatlan de Canas & Maize Amatlan de Canas & 3 \\
\hline Teosinte Amatlan de Canas & Maize Los Guajes & 3 \\
\hline Teosinte Amatlan de Canas & Maize San Lorenzo & 3 \\
\hline Teosinte Amatlan de Canas & Teosinte Los Guajes & 3 \\
\hline Teosinte Amatlan de Canas & Teosinte Palmar Chico & 3 \\
\hline Teosinte Amatlan de Canas & Teosinte San Lorenzo & 3 \\
\hline Teosinte Amatlan de Canas & Teosinte Crucero Lagunitas & 1 \\
\hline Teosinte Amatlan de Canas & Teosinte El Rodeo & 1 \\
\hline Teosinte Crucero Lagunitas & & 9 \\
\hline Teosinte Crucero Lagunitas & Maize Crucero Lagunitas & 6 \\
\hline Teosinte Crucero Lagunitas & Maize Los Guajes & 5 \\
\hline Teosinte Crucero Lagunitas & Teosinte Amatlan de Canas & 4 \\
\hline Teosinte Crucero Lagunitas & Teosinte El Rodeo & 4 \\
\hline Teosinte Crucero Lagunitas & Maize Palmar Chico & 3 \\
\hline
\end{tabular}


bioRxiv preprint doi: https://doi.org/10.1101/2021.09.09.459637; this version posted September 10, 2021. The copyright holder for this preprint (which was not certified by peer review) is the author/funder, who has granted bioRxiv a license to display the preprint in perpetuity. It is made available under aCC-BY 4.0 International license.

\begin{tabular}{|c|c|c|}
\hline Teosinte Crucero Lagunitas & Maize San Lorenzo & 3 \\
\hline Teosinte Crucero Lagunitas & Maize Amatlan de Canas & 1 \\
\hline Teosinte Crucero Lagunitas & Teosinte Palmar Chico & 1 \\
\hline Teosinte Los Guajes & Maize Crucero Lagunitas & 3 \\
\hline Teosinte Los Guajes & Teosinte Amatlan de Canas & 3 \\
\hline Teosinte Los Guajes & & 3 \\
\hline Teosinte Los Guajes & Maize Los Guajes & 1 \\
\hline Teosinte Los Guajes & Maize Palmar Chico & 1 \\
\hline Teosinte Los Guajes & Maize San Lorenzo & 1 \\
\hline Teosinte Palmar Chico & & 8 \\
\hline Teosinte Palmar Chico & Maize Crucero Lagunitas & 5 \\
\hline Teosinte Palmar Chico & Teosinte Amatlan de Canas & 5 \\
\hline Teosinte Palmar Chico & Maize Palmar Chico & 4 \\
\hline Teosinte Palmar Chico & Maize Los Guajes & 3 \\
\hline Teosinte Palmar Chico & Maize San Lorenzo & 3 \\
\hline Teosinte Palmar Chico & Teosinte El Rodeo & 2 \\
\hline Teosinte Palmar Chico & Maize Amatlan de Canas & 1 \\
\hline Teosinte Palmar Chico & Teosinte Crucero Lagunitas & 1 \\
\hline Teosinte San Lorenzo & Maize Crucero Lagunitas & 5 \\
\hline Teosinte San Lorenzo & & 5 \\
\hline Teosinte San Lorenzo & Teosinte Amatlan de Canas & 3 \\
\hline Teosinte San Lorenzo & Maize Palmar Chico & 2 \\
\hline Teosinte San Lorenzo & Teosinte El Rodeo & 2 \\
\hline Teosinte San Lorenzo & Maize Amatlan de Canas & 1 \\
\hline Teosinte San Lorenzo & Maize Los Guajes & 1 \\
\hline Teosinte San Lorenzo & Maize San Lorenzo & 1 \\
\hline
\end{tabular}




\section{Supplement III}

\section{Validating empirical relationship between $\alpha$ and $\theta$.}

To better understand and validate the observed empirical relationship between $\alpha$, the proportion of new mutations fixed by natural selection, and $\theta$, the population scaled mutation rate $\left(4 N_{e} \mu\right.$ ), we conducted simulations using SLiM (Haller and Messer 2019). Each simulation consisted of a 20Mb sequence with constant recombination and mutation rates of $1 \times 10^{6}$ and $3 \times 10^{6}$, respectively, both of which are 100-fold higher than our estimated rates in maize. Matching the average values for the maize genome, the simulated $20 \mathrm{Mb}$ sequence had 300 gene regions separated by $30 \mathrm{~Kb}$ each. Each gene region consisted of four 200bp exons and three $300 \mathrm{bp}$ introns. Mutations were only simulated within exons. Fitness effects of mutations were simulated to be neutral, strictly deleterious, or strictly positive, where the two non-neutral mutation classes were drawn from independent gamma distributions with mean and shape parameters $\left(\mu_{+}\right.$, shape $e_{+}$, and $\mu_{-}$, shape $e_{-}$, for the positive and negative distributions, respectively). Following the empirical ratio of 0 -fold nonsynonymous to 4 -fold synonymous sites, the proportion of non-neutral mutations to neutral mutations was simulated as 3.5 to 1 . The ratio of positively to negatively selected mutations $\left(p_{-}\right)$was allowed to vary across simulations. We implemented as simple 3 -epoch demographic model where the three population sizes ("ancestral" $=N_{A}$, "bottleneck" $=N_{B}$, and "modern" $=N_{0}$ ) and the generation times ("bottleneck" $=T_{B}$ and "modern" $=T_{0}$ ) for changing to a new epoch were allowed to vary across simulations. For each simulation, we computed $\alpha=\frac{\rho_{+}}{\rho_{+}+\rho_{-}+\rho_{0}}$, where $\rho_{+}, \rho_{-}, \rho_{0}$, are the frequency of positive, negative, and neutral fixations that occurred over the course of the simulation. Simulations were always initiated with a biurn-in of $10 * N_{A}$ generations to ensure simulations were near equilibrium conditions prior to implementing demographic changes. To make direct comparisons with the empirical data, we converted the demographic parameters to a single value of $\theta$ as the harmonic mean of the three population size parameters, weighted by the number of generations spent at that population size. This resulted in 7 free parameters that were drawn from uniform priors (Table S3). From 1000 simulations, we fit a linear model predicting $\alpha$ from the free parameters.

As expected from theory, we found a positive effect of the population scaled mutation rate $\left(\theta=4 N_{e} \mu\right)$ on $\alpha$ (Table S3 and Figure S2), and the total number of adaptive substitutions (Table S4 and Figure S2). However, in both cases, the relationship appears to be nonlinear and plateaus for higher values of $\theta$. The non-linearity persists after accounting for the effect of other parameters (Figure S2 C and D). Nonetheless, $\alpha$ and the number of adaptive substitutions increase over the broad range of $\theta$ simulated.

Unsurprisingly, the mean of both positive and negative fitness distributions, and the proportion of non-neutral mutations simulated as deleterious, were also all significant predictors of $\alpha$. As noted in the discussion, given the relative ease of fixing strongly adaptive mutations, it is now widely believed that difference in the ability to purge weakly deleterious alleles plays an important role in determining $\alpha$. Our simulations were conducted over a broad distribution of fitness effects. As such, we cannot directly identify to what extent the relationship between $\alpha$ and $\theta$ is driven by increasing efficacy of larger populations to fix positive mutations versus their ability to purge weekly deleterious ones. However, based on our simulations, it seems likely that both phenomena contribute to $\alpha$, though which is more important will depend on the particular combination of parameters that a population occupies.

Table S3 Regression predicting $\alpha$ from demographic and fitness effect parameters. The Prior column contains the distributions that each parameter was simulated from. The remaining parameters are those output from the $l m$ function in $R$. For each simulation, $\theta$ was calculated as the harmonic mean of the three population size parameters, weighted by the number of generations spent at that population size. Priors for the two population size change parameters $T_{0}$ and $T_{B}$ were drawn from a uniform with bound 0 and $N_{A}$, but these values were added on the the previous number of generations, such that the total number of generations was $10 N_{A}+T_{B}+T_{0}$

\begin{tabular}{lccccr}
\hline Parameter & Prior & Estimate & Std. Error & $\mathrm{t}$ value & $\operatorname{Pr}(>|\mathrm{t}|)$ \\
\hline \hline (Intercept) & $\mathrm{N} / \mathrm{A}$ & 3.779 & 0.197 & 19.151 & $1.13 \mathrm{e}-58$ \\
$\theta$ & $\mathrm{N} / \mathrm{A}$ & 16.641 & 1.288 & 12.923 & $2.99 \mathrm{e}-32$ \\
$N_{B}$ & $U(10,2000)$ & $\mathrm{N} / \mathrm{A}$ & $\mathrm{N} / \mathrm{A}$ & $\mathrm{N} / \mathrm{A}$ & $\mathrm{N} / \mathrm{A}$ \\
$N_{A}$ & $U(10,2000)$ & $\mathrm{N} / \mathrm{A}$ & $\mathrm{N} / \mathrm{A}$ & $\mathrm{N} / \mathrm{A}$ & $\mathrm{N} / \mathrm{A}$ \\
$N_{0}$ & $U(55,2000)$ & $\mathrm{N} / \mathrm{A}$ & $\mathrm{N} / \mathrm{A}$ & $\mathrm{N} / \mathrm{A}$ & $\mathrm{N} / \mathrm{A}$ \\
$T_{B}$ & $U\left(0, N_{A}\right)$ & $\mathrm{N} / \mathrm{A}$ & $\mathrm{N} / \mathrm{A}$ & $\mathrm{N} / \mathrm{A}$ & $\mathrm{N} / \mathrm{A}$ \\
$T_{0}$ & $U\left(0, N_{A}\right)$ & $\mathrm{N} / \mathrm{A}$ & $\mathrm{N} / \mathrm{A}$ & $\mathrm{N} / \mathrm{A}$ & $\mathrm{N} / \mathrm{A}$ \\
$p_{-}$ & $U(0.9,1)$ & -3.999 & 0.207 & -19.314 & $1.83 \mathrm{e}-59$ \\
$\mu_{+}$ & $U(0,0.05)$ & 5.633 & 0.454 & 12.394 & $3.75 \mathrm{e}-30$ \\
shape $_{+}$ & $U(0,1)$ & 0.063 & 0.020 & 3.147 & 0.00177 \\
$\mu_{-}$ & $U(-0.05,0)$ & -0.984 & 0.386 & -2.546 & 0.0113 \\
shape $_{-}$ & $U(0,1)$ & 0.125 & 0.019 & 6.457 & $3.04 \mathrm{e}-10$ \\
\hline
\end{tabular}


bioRxiv preprint doi: https://doi.org/10.1101/2021.09.09.459637; this version posted September 10, 2021. The copyright holder for this preprint (which was not certified by peer review) is the author/funder, who has granted bioRxiv a license to display the preprint in perpetuity. It is made available under aCC-BY 4.0 International license.

Table S4 Regression predicting the count of substitutions $\alpha_{\text {substitutions }}$ (as opposed to the proportion of adaptive substitutions in Table S3) from demographic and fitness effect parameters.

\begin{tabular}{lcccc}
\hline Parameter & Estimate & Std. Error & $\mathrm{t}$ value & $\operatorname{Pr}(>|\mathrm{t}|)$ \\
\hline \hline (Intercept) & 13.311 & 0.918 & 14.493 & $1.13 \mathrm{e}-38$ \\
$\theta$ & 120.936 & 6.045 & 20.006 & $1.69 \mathrm{e}-62$ \\
$p_{-}$ & -11.817 & 0.972 & -12.159 & $3.14 \mathrm{e}-29$ \\
$\mu_{+}$ & 17.709 & 2.134 & 8.300 & $1.55 \mathrm{e}-15$ \\
shape $_{+}$ & 0.246 & 0.094 & 2.613 & 0.0093 \\
$\mu_{-}$ & 4.686 & 1.814 & 2.583 & 0.0102 \\
shape $_{-}$ & -0.023 & 0.091 & -0.259 & 0.796 \\
\hline
\end{tabular}

A

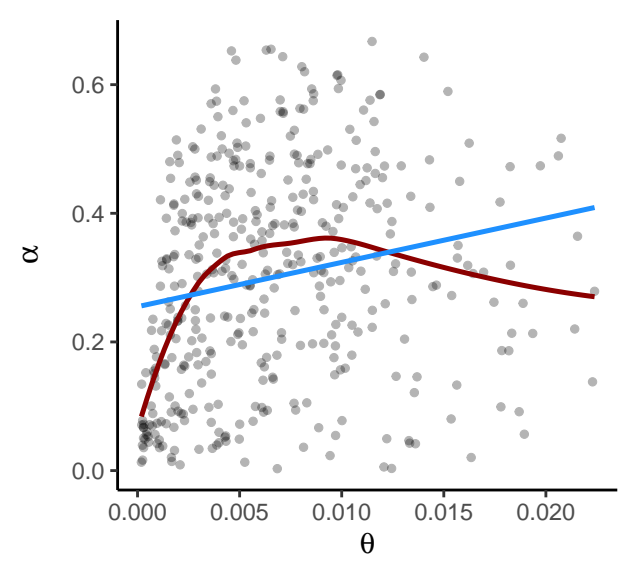

C

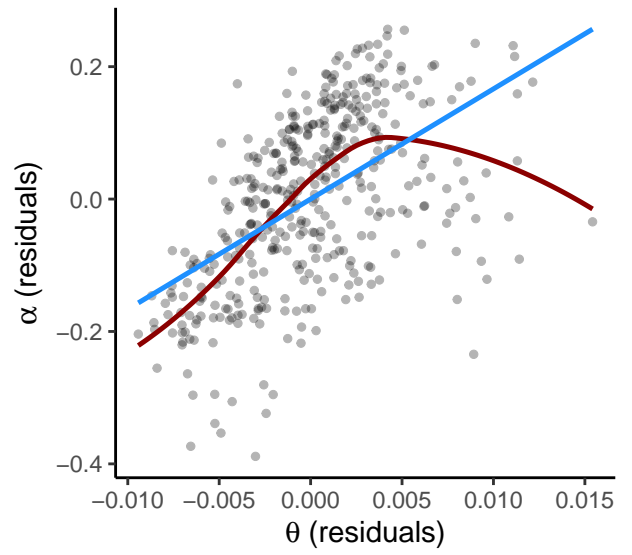

B

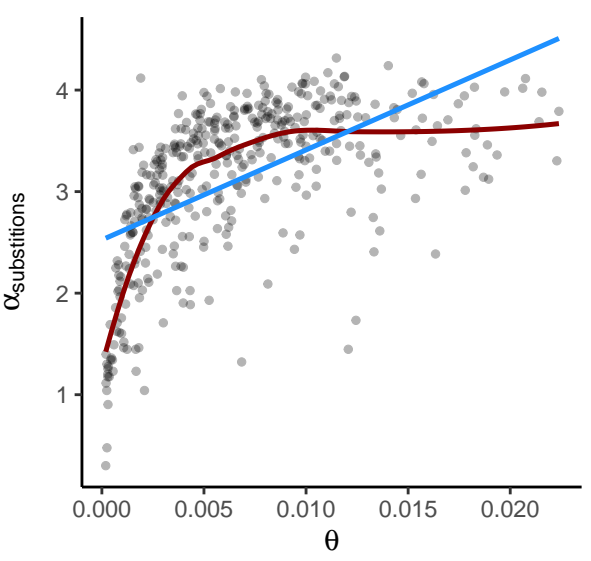

D

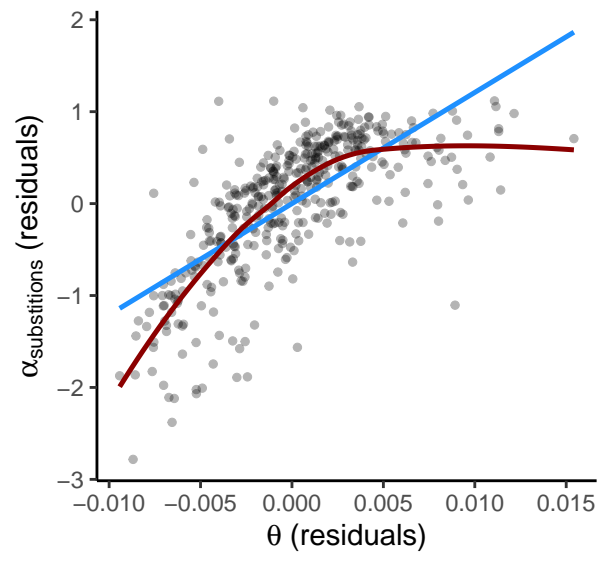

Figure S2 population scaled mutation rate $\left(\theta=4 N_{e} \mu\right)$ is associated with $\alpha$ over a broad range of demography and fitness parameters. The raw relationship between $\theta$ and $\alpha$ (A) and $\theta$ and the count of adaptive substitions $\left(\alpha_{\text {substitutions }}\right)$ (B) from simulations, not taking account any associations between $\alpha$ and the other parameters or $\theta$ and the other parameters. Added variable (or partial regression) plots showing the relationship between model residuals of $\theta$ and $\alpha$ (C) and and $\theta$ and $\alpha_{\text {substitutions }}$ (D), which take account of the associations between both parameters and the other model predictors (see table S3.) Blue lines for each panel show the linear model fit between the $x$ and $y$ variables, while the dark red lines show the loess smooth fit. 
bioRxiv preprint doi: https://doi.org/10.1101/2021.09.09.459637; this version posted September 10, 2021. The copyright holder for this preprint (which was not certified by peer review) is the author/funder, who has granted bioRxiv a license to display the preprint in perpetuity. It is made available under aCC-BY 4.0 International license.

\section{Supplement IV Predicting $\alpha$ by mutation type}

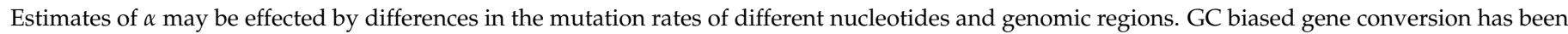

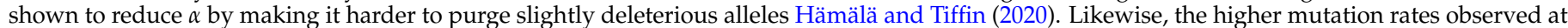

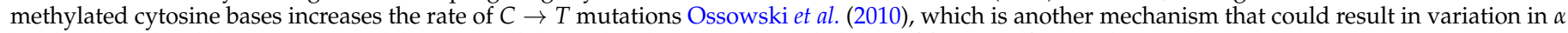
by changing the ability to purging deleterious alleles, or by changing the probability of fixation of new adaptive mutations.

To study this, we used the same approach as Hämälä and Tiffin (2020), where we separated the site frequency spectra based on mutation types

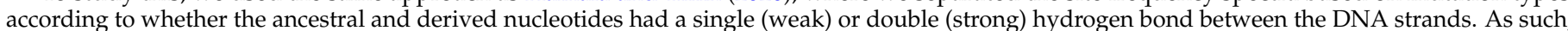
we studied three mutations types: $A / T \rightarrow G / C$ mutations (WS), $G / C \rightarrow A / T(S W)$ and $C / G \rightarrow G / C$ or $A / T \rightarrow T / A$ (SS_WW).

Unlike patterns found in Arabidopsis (Hämälä and Tiffin 2020), $\alpha$ was highest for WS mutations, although there was considerable overlap between the credible intervals for all mutation types.

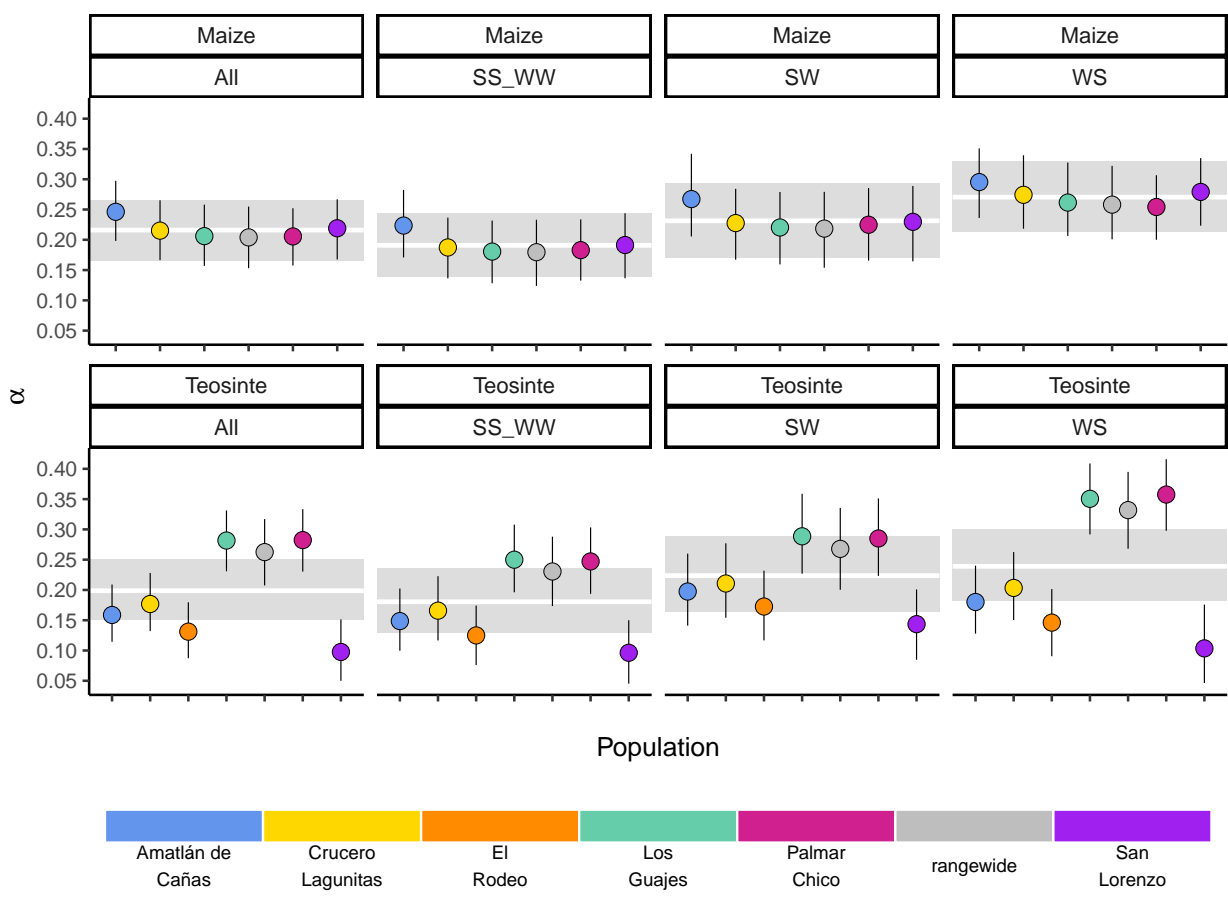

Figure S3 Predicted values of $\alpha$ across mutation types. Grey bands for each mutation type show the $95 \%$ credible intervals averaged over each population. 
bioRxiv preprint doi: https://doi.org/10.1101/2021.09.09.459637; this version posted September 10, 2021. The copyright holder for this preprint (which was not certified by peer review) is the author/funder, who has granted bioRxiv a license to display the preprint in perpetuity. It is made available under aCC-BY 4.0 International license.

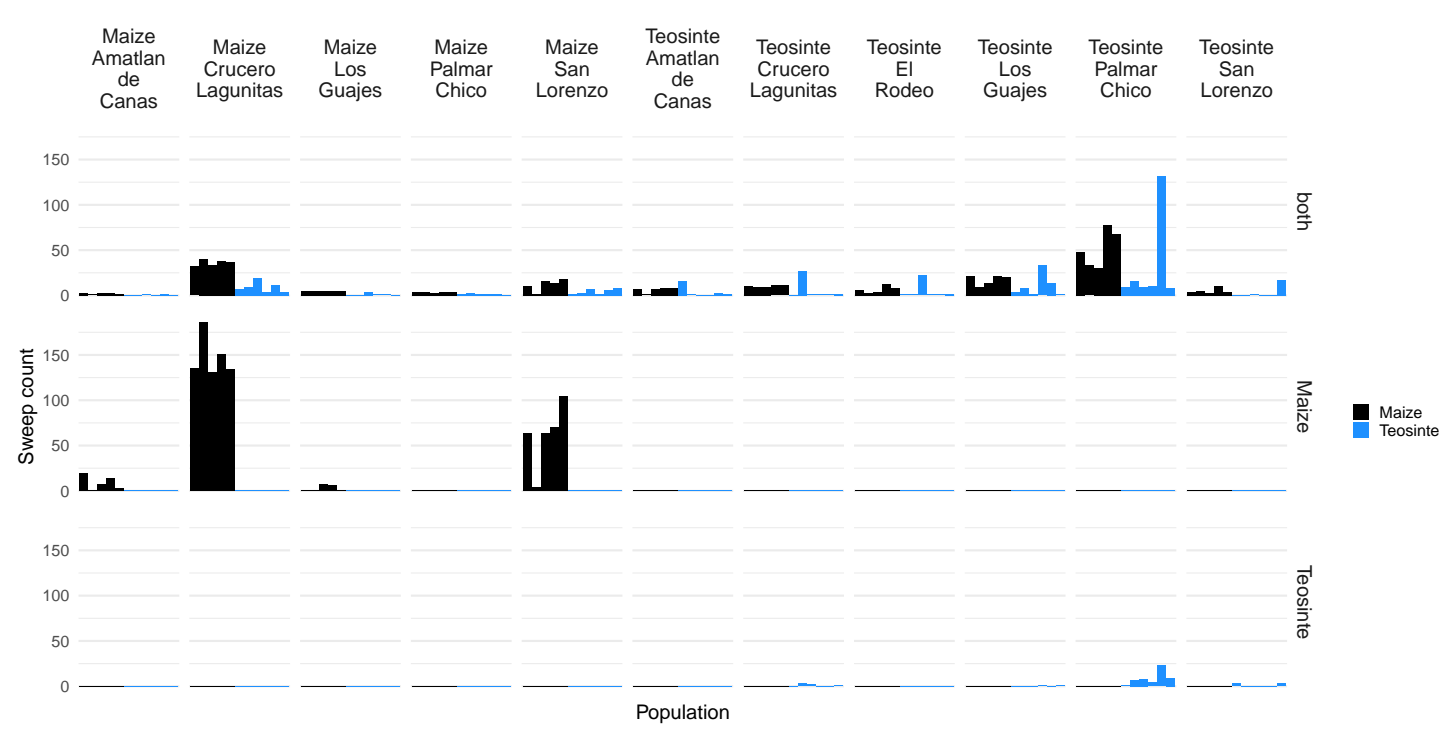

Figure S4 Frequency of each population as the mutation source for sweeps shared via migration. The order of populations along the $\mathrm{x}$ axis matches that of the source populations labeled for each strip along the top.

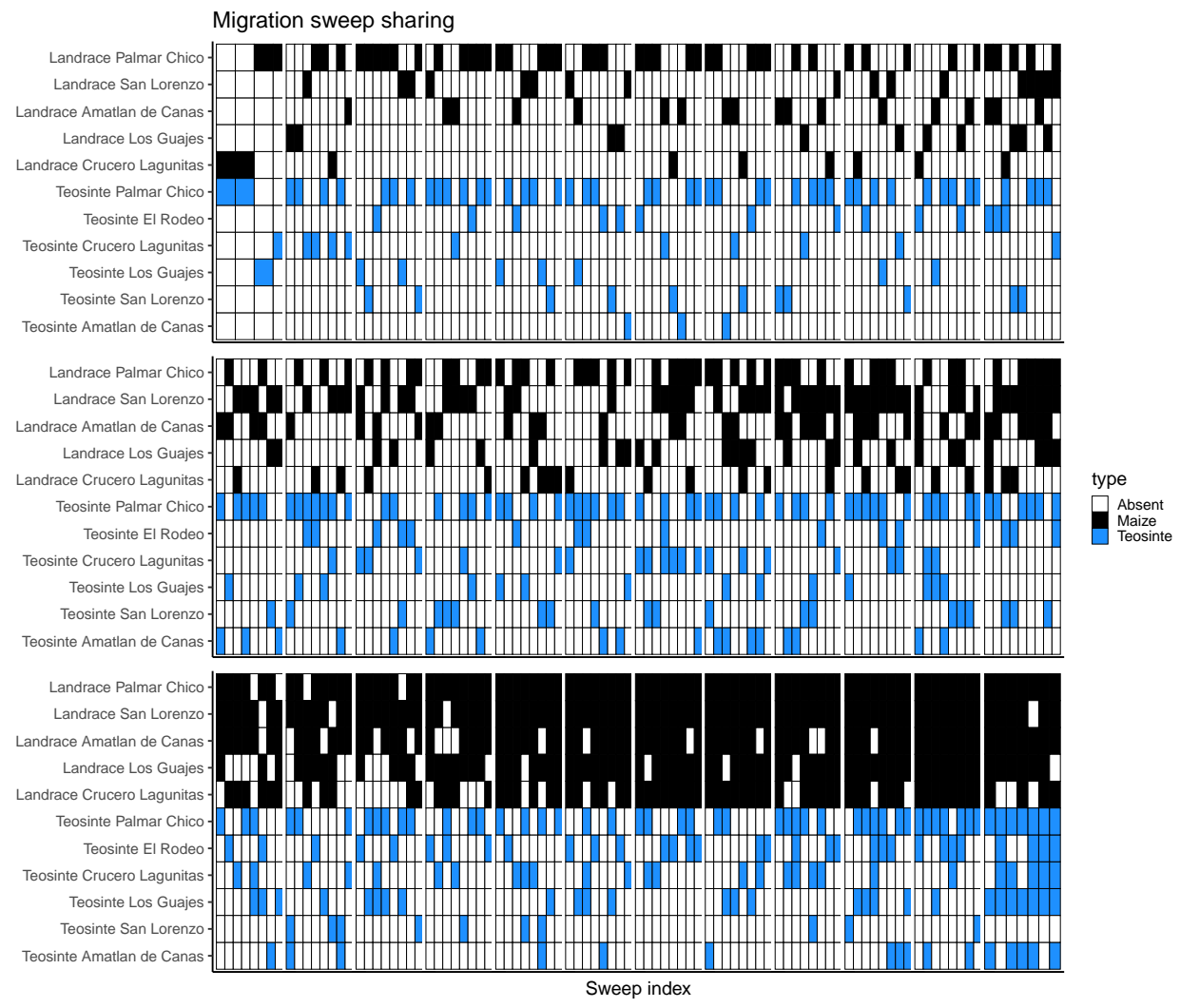

Figure S5 Complete accounting of inferred sweeps shared via migration. The $x$ axis is sorted by the number of populations each sweep was found in. Populations are sorted along the y axis first by subspecies then by their number of sweeps. 
bioRxiv preprint doi: https://doi.org/10.1101/2021.09.09.459637; this version posted September 10, 2021. The copyright holder for this preprint (which was not certified by peer review) is the author/funder, who has granted bioRxiv a license to display the preprint in perpetuity. It is made available under aCC-BY 4.0 International license.

\section{Demystifying the high proportion of sweeps unique to the rangewide maize sample}

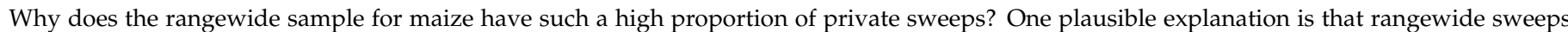

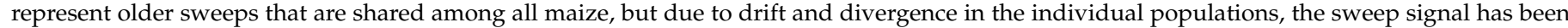

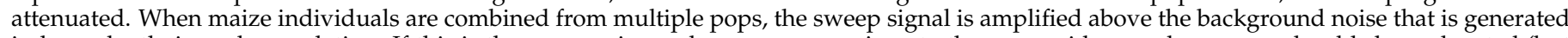

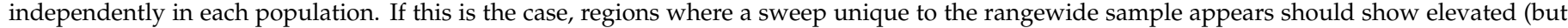
perhaps not statistically significant) evidence of a sweep in the individual populations.

We confirmed this hypothesis by comparing the distribution of the RAiSD $\mu$ statistic within the regions we identified as private sweeps in the

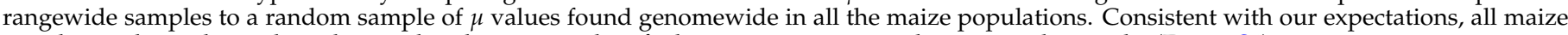
populations have elevated $\mu$ values within the regions identified as private sweeps in the rangewide sample. (Figure S6).

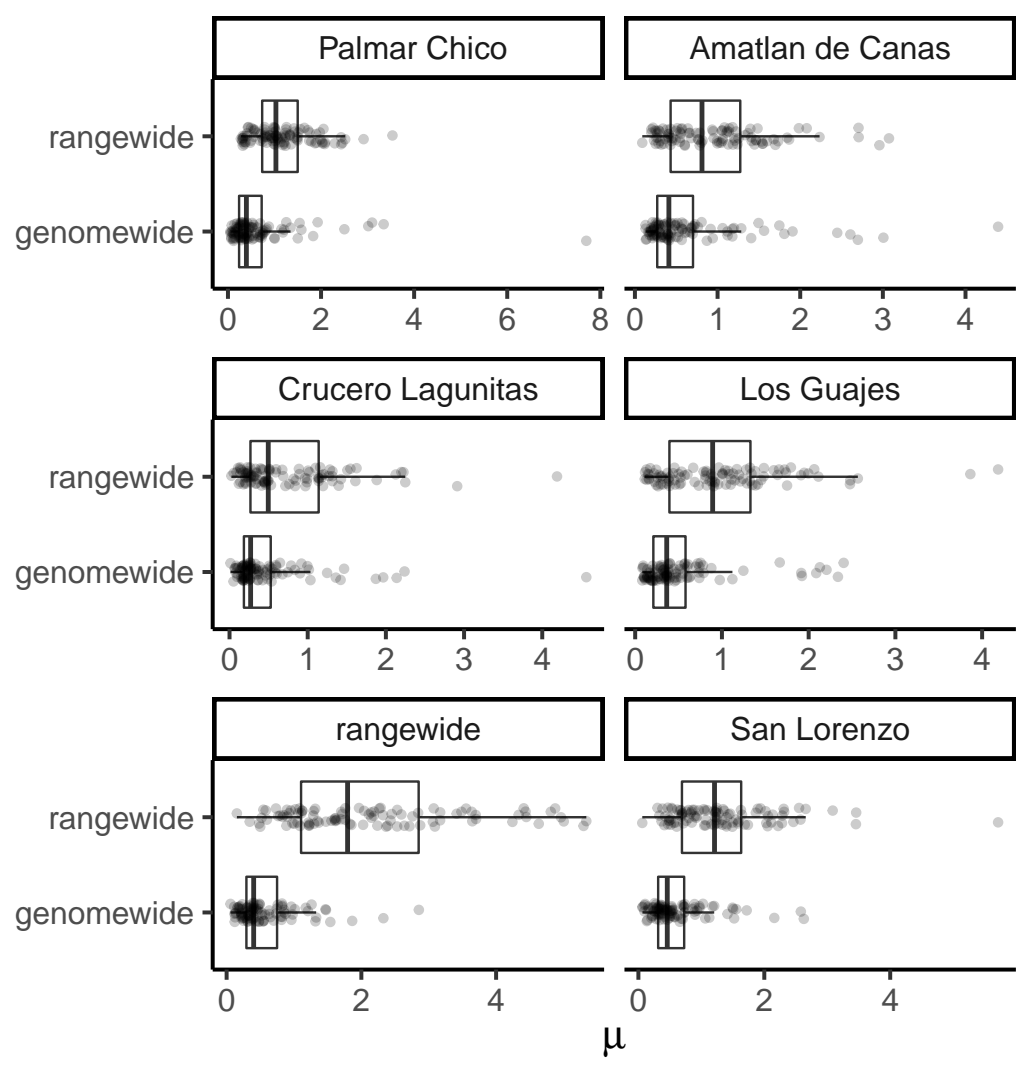

Figure S6 The distribution of RAiSD $\mu$ statistics within sweep regions unique to the rangewide sample for all maize populations. For each population, we randomly sampled $100 \mu$ values from within the rangewide sweep regions and genomewide. 